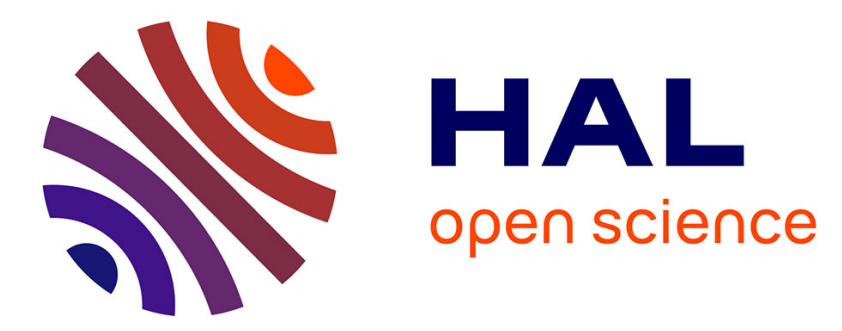

\title{
Approximation of non-Lipschitz SDEs by Picard iterations
}

\author{
Julien Baptiste, Julien Grepat, Emmanuel Lépinette
}

\section{To cite this version:}

Julien Baptiste, Julien Grepat, Emmanuel Lépinette. Approximation of non-Lipschitz SDEs by Picard iterations. Applied Mathematical Finance, 2018, 25(2018) (2), pp.148-179. hal-01397399v2

\section{HAL Id: hal-01397399 \\ https://hal.science/hal-01397399v2}

Submitted on 28 Aug 2018

HAL is a multi-disciplinary open access archive for the deposit and dissemination of scientific research documents, whether they are published or not. The documents may come from teaching and research institutions in France or abroad, or from public or private research centers.
L'archive ouverte pluridisciplinaire HAL, est destinée au dépôt et à la diffusion de documents scientifiques de niveau recherche, publiés ou non, émanant des établissements d'enseignement et de recherche français ou étrangers, des laboratoires publics ou privés. 


\title{
Approximation of non-Lipschitz SDEs by Picard iterations.
}

\author{
Julien Baptiste ${ }^{1}$ Julien GREPAT $^{1}$ \\ Emmanuel LEPINETTE ${ }^{2}$ \\ ${ }^{1}$ Paris-Dauphine University, PSL National Research, Ceremade, CNRS, Place du \\ Maréchal De Lattre De Tassigny, 75775 Paris cedex 16, France. \\ Emails: julien.baptiste@ceremade.dauphine.fr; julien.grepat@gmail.com. \\ ${ }^{2}$ Paris-Dauphine University, PSL National Research, Ceremade, CNRS, Place du \\ Maréchal De Lattre De Tassigny, 75775 Paris cedex 16, France and \\ Gosaef, Faculté des Sciences de Tunis, Manar II-Tunis, Tunisia. \\ Email: emmanuel.lepinette@ceremade.dauphine.fr
}

\begin{abstract}
In this paper, we propose an approximation method based on Picard iterations deduced from the Doléans-Dade exponential formula. Our method allows to approximate trajectories of Markov processes in a large class, e.g. solutions to non-Lipchitz SDEs. An application to the pricing of Asian-style contingent claims in the CEV model is presented and compared to other methods of the literature.
\end{abstract}

Keywords and phrases: Pricing, European Options

MSC: 60G44, G11-G13.

\section{Introduction}

In frictionless models without arbitrage opportunities, it is possible to define the price of a contingent claim as the initial value of the unique portfolio process whose terminal value coincides with the payoff, see [4, Section 3.2.2]. The price of a replicable contingent claim may be deduced as an expectation as soon as the price process is a local martingale and the replicating portfolio is a martingale under some equivalent probability measure. If the 
price dynamics is simple enough, e.g. in the Black and Scholes model, it is possible to obtain an explicit expression for the Call or Put option prices. When sophisticated models are considered for a sake of realism $[3,7,11]$, one can not expect to compute easily option prices which do not admit pricing formulas in closed form. For this purpose, Monte-Carlo methods have been widely developed to simulate trajectories of diffusion processes and deduce approximations of European option prices [2, 17].

An alternative way is to study the density function of the terminal price value as in the Black and Scholes model [1]. This is also possible for the CEV model [18] where analytic expressions of European Call and Put option prices are deduced. Nevertheless, it is not possible to evaluate the price of an Asian option since a knowledge of the whole trajectory is required. Moreover, the Monte Carlo discretization method seems to be useless as the diffusion coefficient is not Lipschitz so that the Euler scheme should explode. In $[9,16]$, the asset price process is alternatively approximated through a Picard iteration scheme followed by approximations based on Wiener-Ito chaos expansions. Only the first three terms in the expansions are considered as significative and the associated density functions are approximated. The accuracy of the suggested numerical method illustrated by numerical implementations is highlighted even if it is not confirmed by mathematical arguments. A direct discretization scheme based on Doléans-Dade formula is also used (without mathematical proof of the convergence) in [20] to simulate trajectories in the CEV model. Inspired by these works, our goal is to study the method based on Picard iterations to approximate Asian option prices when the risky asset dynamics is a stochastic differential equation with non Lipschitz-coefficients.

The outline of our work is as follows. By a truncation, we first approximate the stochastic process of interest by a diffusion process with Lipschitz coefficients. Second, we introduce a Picard iteration technique that approximates the process as a Doléans-Dade exponential. Finally, a Monte Carlo discretization scheme is implemented. Comparatively to the classical Euler scheme, we obtain a convergence order $n$ instead of $\sqrt{n}$ where $n$ is the number of discrete 
dates. The method may be applied to the pricing of a large class of European and Asian options. We illustrate it by computing prices of average Put options in the CEV model where the diffusion coefficient is not Lipschitz. Comparing with the exact formulas and other techniques, our method appears to provide accurate results. Moreover, as soon as we consider an average Put option with more than three dates, our method is less time-consuming than the method combining Monte Carlo simulations and analytic expressions. Nevertheless, in some cases, a simple truncated Euler scheme appears to be rather efficient whereas the direct discretization scheme based on the Doléans-Dade formula without truncation does not converge. For some other cases, e.g. the CEV model with $\beta>1$, we clearly observe that the simple Euler scheme explodes but, in that case the backward Euler-Maruyama scheme is the most efficient.

\section{Model}

\subsection{Notations}

We denote by $\mathbf{R}$ the set of all real numbers.

The smallest $\sigma$-algebra containing a family of subsets $\mathcal{A}$ is denoted by $\sigma(\mathcal{A})$. The set of all bounded real-valued functions on $[0, T]$ is denoted by $\mathcal{B}([0, T])$. Let $(\Omega, \mathcal{F}, P)$ be a stochastic basis with a complete filtration $\mathcal{F}=\left(\mathcal{F}_{t}\right)_{t \in[0, T]}$ satisfying the usual hypothesis and $T>0$ is fixed. We consider a standard Brownian motion $W$ adapted to $\mathcal{F}$. For any predictable process $H$, we consider the stochastic integral of $H$ with respect to $W$ denoted by

$$
H \cdot W_{t}:=\int_{0}^{t} H_{u} d W_{u}, \quad t \in[0, T],
$$

when existence holds. The Doleans-Dade exponential is denoted by

$$
\mathcal{E}_{t}(H)=\exp \left(H \cdot W_{t}-\frac{1}{2} \int_{0}^{t} H_{u}^{2} d u\right), \quad t \in[0, T] .
$$

In the following, $S_{0}$ is the deterministic initial value at time 0 of a process $S$ modelling an asset price in a financial market model. In the following, 
we consider uniform partitions $\left(\tau_{n}\right)_{n \geq 0}$ of the interval $[0, T]$. The grid $\tau_{n}$ is composed of $n+1$ dates where $n$ is called the order of the discretization scheme. It is defined by

$$
\tau_{n}=\left\{t_{0}=0, t_{1}=T / n, t_{2}=2 T / n, \cdots t_{n}=T\right\} .
$$

We use the abuse of notation $t_{i}^{n}=t_{i}=(T / n) i, i=0, \cdots, n$, for a sake of simplicity. If $X$ is a stochastic process defined on $[0, T]$, we denote by $X^{(n)}$ the piecewise constant process defined by

$$
X_{t}^{(n)}=X_{t_{i}}, \quad t \in\left[t_{i}, t_{i+1}\right), \quad X_{T}^{(n)}=X_{T}
$$

\subsection{The stochastic differential equation}

Let $\left(\Omega, \mathcal{F}, \mathbb{F}=\left(\mathcal{F}_{t}\right)_{t \in[0, T]}, \mathrm{P}\right)$ be a continuous-time stochastic basis satisfying the usual assumptions and supporting a standard Brownian motion $W$, i.e. $\mathcal{F}_{t}:=\sigma\left(W_{s}: s \leq t\right) \vee \mathcal{N}$ where $\mathcal{N}$ designates the P-negligible sets. In the case of a financial market model, there is a risk-free bond given by the interest rate $r^{0}$ and a risky asset price stochastic process $S$.

By [8, Theorem $2.2 \mathrm{p} \mathrm{104]}$, recall that we have the following.

Proposition 2.1. Let $\sigma:[0, T] \times \mathbf{R} \mapsto \mathbf{R}$ and $r:[0, T] \times \mathbf{R} \mapsto \mathbf{R}$ be Lipschitz functions. The stochastic differential equation (s.d.e.)

$$
d S(t)=S(t) \sigma(t, S(t)) d W_{t}+S(t) r(t, S(t)) d t, \quad S(0)=S_{0}>0
$$

admits a unique strong solution $S$. Moreover, it is positive.

In the sequel, we shall consider locally Lipschitz coefficients $r$ and $\sigma$ and we assume that $r$ is bounded. We suppose that $S=0$ on $[\tau \wedge T, T]$ where $\tau:=\inf \left\{t: S_{t}=0\right\}$, meaning that 0 is an absorbing point. The s.d.e. (2.1) admits a unique non negative solution under the conditions of the proposition above but we only suppose one of the following Conditions 2.2 or 2.3. 
Condition 2.2. There exists constants $C_{\kappa}>$ for every $\kappa>0$, such that

$$
\begin{aligned}
& |\sigma(t, x \wedge \kappa)-\sigma(u, x \wedge \kappa)| \leq C_{\kappa}|t-u|, \quad(t, u, x) \in\left(\mathbf{R}_{+}\right)^{2} \times(0, \infty) \\
& |r(t, x \wedge \kappa)-r(u, x \wedge \kappa)| \leq C_{\kappa}|t-u|, \quad(t, u, x) \in\left(\mathbf{R}_{+}\right)^{2} \times(0, \infty) \\
& |\sigma(t, x \wedge \kappa)-\sigma(t, y \wedge \kappa)| \leq C_{\kappa}|x-y|, \quad(t, x, y) \in \mathbf{R}_{+} \times(0, \infty)^{2} \\
& |r(t, x \wedge \kappa)-r(t, y \wedge \kappa)| \leq C_{\kappa}|x-y|, \quad(t, x, y) \in \mathbf{R}_{+} \times(0, \infty)^{2} \\
& |r(t, x \wedge \kappa)|+|\sigma(t, x \wedge \kappa)| \leq C_{\kappa}, \quad(t, x, y) \in \mathbf{R}_{+} \times(0, \infty)^{2}
\end{aligned}
$$

Condition 2.3. There exists constants $C_{\kappa}>$ for every $\kappa>0$, such that

$$
\begin{aligned}
& |\sigma(t, x \vee \kappa)-\sigma(u, x \vee \kappa)| \leq C_{\kappa}|t-u|, \quad(t, u, x) \in\left(\mathbf{R}_{+}\right)^{2} \times(0, \infty), \\
& |r(t, x \vee \kappa)-r(u, x \vee \kappa)| \leq C_{\kappa}|t-u|, \quad(t, u, Y) \in\left(\mathbf{R}_{+}\right)^{2} \times(0, \infty), \\
& \left|\sigma\left(t, e^{X} \vee \kappa^{-1}\right)-\sigma\left(t, e^{Y} \vee \kappa^{-1}\right)\right| \leq C_{\kappa}|X-Y|, \quad(t, X, Y) \in \mathbf{R} \times \mathbf{R}^{2}, \\
& \left|r\left(t, e^{X} \vee \kappa^{-1}\right)-r\left(t, e^{Y} \vee \kappa^{-1}\right)\right| \leq C_{\kappa}|X-Y|, \quad(t, X, Y) \in \mathbf{R} \times \mathbf{R}^{2}, \\
& |r(t, x \vee \kappa)|+|\sigma(t, y \vee \kappa)| \leq C_{\kappa}, \quad(t, x, y) \in \mathbf{R}_{+} \times(0, \infty)^{2} .
\end{aligned}
$$

Moreover, when $P(\tau<T)>0$, we suppose that the mappings $x \mapsto \sigma(t, x)$ and $x \mapsto r(t, x)$ are differentiable on $(0, \infty)$ for all $t \in[0, T]$ and $x \nabla_{x} \sigma(t, x)$, $x \nabla_{x} r(t, x)$ are bounded on $\left[\kappa^{-1}, \infty\right)$ by $C_{\kappa}$.

Remark 2.4. Under Condition 2.2, S admits moments at any order, see the proof in Section 8. Notice that this assumption is satisfied by the CEV model $d S_{t}=\sigma S_{t}^{\beta} d W_{t}$ and, more generally, when $\sigma$ is of the form $\sigma(t, x)=$ $\nu(t) x^{\beta(t)-1}, x>0$, where $\nu$ and $\beta \geq 1$ are bounded functions and $\nu$ is Lipschitz.

Condition 2.3 is satisfied by the CEV model, e.g. when $\sigma$ is of the form $\sigma(t, x)=\nu(t) x^{\beta(t)-1}$ where $\nu$ is Lipschitz and bounded and $1-\alpha \leq \beta \leq 1$, $\alpha \in \mathbf{R}_{+}$. This also includes the CIR model and more generally mean-reverting SDEs as used in finance, see numerical simulations below.

In finance, under a risk-neutral probability measure for $S$, e.g. when the market is complete, we are interested in approximating the price of a contingent claim whose payoff $G(S) 1_{\tau>T}$ depends on the whole trajectory $S=\left(S_{t}\right)_{t \in[0, T]}$. 
Note that the delivered payoff $G(S) 1_{\tau>T}$ is 0 when $S_{T}=0$, i.e. when the firm having issued the risky asset failed.

We suppose that the functional $G: \mathcal{B}([0, T]) \rightarrow \mathbb{R}_{+}$satisfies the Lipschitz condition:

$$
|G(\alpha)-G(\beta)| \leq C|| \alpha-\beta \|, \quad \alpha, \beta \in \mathcal{B}([0, T]),
$$

where $C>0$ and $\|\cdot\|$ is the norm of uniform convergence on $[0, T]$, i.e.

$$
\|f\|:=\sup _{t \in[0, T]}|f(t)|, \quad f \in \mathcal{B}([0, T]) .
$$

If we assume that $\mathrm{P}$ is the unique risk-neutral probability measure in a complete market, the smallest price $v$ of the contingent claim $G(S) 1_{\tau>T}$ is evaluated under $\mathrm{P}$ by $v=\mathbf{E} e^{-r^{0} T} G(S) 1_{\tau>T}$ where $\mathbf{E}$ is the expectation under $\mathrm{P}$ and $r^{0}$ is the constant risk-free interest rate.

In the following, a stochastic process $r: u \mapsto r_{u}$ models an interest rate of the market. The price of the associated bond with initial value 1 is

$$
F_{t}(r):=\exp \left(\int_{0}^{t} r_{u} d u\right), \quad t \in[0, T]
$$

We consider a class of payoff functions $G$ adapted to the numerical scheme we propose. For a sake of simplicity, we make the abuse of notation $r_{u}=r\left(u, S_{u}\right)$ as well as $\sigma_{u}=\sigma\left(u, S_{u}\right)$ so that the notations $F(r)$ and $\mathcal{E}(\sigma)$ make sense.

Definition 2.5. We say that the payoff function $G$ is adapted to the discretization scheme defined by the partition $\tau_{n}$ if there exists $\alpha(n) \geq 1, p, q \in \mathbb{N}$ such that, for all Lipschitz functions $\sigma$ and $r$ satisfying

$$
\int_{0}^{T} \mathbf{E} F_{u}^{2 p}(r) \mathcal{E}_{u}^{2 p}(\sigma) d u<\infty
$$

we have, for some function $f$,

$$
\left|\mathbf{E} G\left(S_{r, \sigma}\right)-\mathbf{E} G\left(S_{r, \sigma}^{(n)}\right)\right| \leq \frac{f(G, \sigma, r, p, q)}{\alpha(n)}\left(\int_{0}^{T}\left[1+\mathbf{E} S_{r, \sigma}^{2 p}(u) d u\right)^{1 / q},\right.
$$

where $S_{r, \sigma}=F(r) \mathcal{E}(\sigma)$ and $f$ depends on $\sigma, r, p$ through the quantities $\max _{x}|\sigma(t, x) x|^{p} /\left(1+|x|^{2 p}\right)<\infty$ and $\max _{x}|r(t, x) x|^{p} /\left(1+|x|^{2 p}\right)<\infty$. 
Recall that $F(r)$ is given by (2.3) and, in the definition above, the process $S_{r, \sigma}=F(r) \mathcal{E}(\sigma)$ is interpreted as a price. Observe that the European call payoff function is adapted to the discretization whatever $\alpha(n)$ is. Indeed, the payoff function of the European call option with strike $K$ is $G(S)$ where $G(\alpha)=(\alpha(T)-K)^{+}, \alpha \in \mathcal{B}([0, T])$ and, since $G$ only depends on the terminal value of $\alpha$, we have $G\left(S_{r, \sigma}\right)=G\left(S_{r, \sigma}^{(n)}\right)$.

The payoff of the average strike Asian call option with strike $K$ is $G(S)$ where

$$
G(\alpha)=\left(\frac{1}{T} \int_{0}^{T} \alpha(t) d t-K\right)^{+}, \quad \alpha \text { is } \mathcal{B}([0, T])-\text { measurable. }
$$

Lemma 2.6. The average strike Asian call option function $G$ with strike $K$ is adapted to the discretization with $\alpha(n)=n$.

All the proofs are postponed to Section 8.

\section{Approximation of the stochastic process}

The approximation scheme we propose is obtained in three steps. By a truncation, we first approximate the price process by a diffusion process $S_{\kappa}$, $\kappa>0$, with bounded and Lipschitz volatility. By Picard iterations, a recursive scheme is introduced to approximate $S_{\kappa}$ by $S_{\kappa, m}, m \geq 1$, Doléans-Dade solutions to stochastic differential equations defined by iterated and uniformly bounded volatilities. At last, we discretize $S_{\kappa, m}$ in time.

\subsection{Volatility truncation}

Let $\kappa \in \mathbf{R}_{+}$be such that $\kappa \geq \sup _{(t, x) \in \mathbf{R}^{2}} r(t, x)$ and $\kappa>S_{0} \vee 1$ when Condition 2.2 holds and $\kappa^{-1}<\min \left(\max r, S_{0} \vee 1\right)$ when Condition 2.3 is satisfied. Consider $Y_{\kappa}$ the unique solution to the s.d.e.

$$
\begin{aligned}
Y_{\kappa}(0) & =0 \\
d Y_{\kappa}(t) & =\sigma_{\kappa}\left(t, S_{0} e^{Y_{\kappa}(t)}\right) d W_{t}+\gamma_{\kappa}\left(t, S_{0} e^{Y_{\kappa}(t)}\right) d t
\end{aligned}
$$


where $\sigma_{\kappa}(u, x):=\sigma(u, x \wedge \kappa)$ and $r_{\kappa}(u, x):=r(u, x \wedge \kappa)$ when Condition 2.2 holds. If Condition 2.3 holds, we define $\sigma_{\kappa}(u, x):=\sigma\left(u, x \vee \kappa^{-1}\right)$ and $r_{\kappa}(u, x):=r\left(u, x \vee \kappa^{-1}\right)$. At last, we define $\gamma_{\kappa}(t, x)=r_{\kappa}(t, x)-\frac{1}{2} \sigma_{\kappa}^{2}(t, x)$. Observe that $S_{\kappa}:=S_{0} e^{Y_{\kappa}}$ satisfies, for all $t \in[0, T]$,

$$
\begin{aligned}
S_{\kappa}(0) & =S_{0} \\
S_{\kappa}(t) & :=S_{0} \exp \left[\int_{0}^{t} \sigma_{\kappa}\left(u, S_{\kappa}(u)\right) d W_{u}+\int_{0}^{t} \gamma_{\kappa}\left(u, S_{\kappa}(u)\right) d u\right] .
\end{aligned}
$$

Lemma 3.1. Suppose that Conditions 2.2 and 2.3 hold respectively. Then, $S_{\kappa}$ converges pointwise to $S$ as $\kappa \rightarrow \infty$ on the interval $[0, T]$ (on the interval $[0, \tau)$ respectively).

In the following, we use the following assumption:

Condition 3.2. There exists $p>0$ and a constant $C_{p}$ such that

$$
\mathbf{E} \sup _{t \in[0, T]} S_{t}^{2 p}+\sup _{\kappa} \mathbf{E} \sup _{t \in[0, T]} S_{\kappa}^{2 p}(t) \leq C_{p}, \quad \forall \kappa>0
$$

By the Lemmas 3.4 and 3.5 proven in appendix, we deduce that Condition 3.2 is satisfied for the large class of models satisfying the following Condition 3.3 above even if the coefficients of (2.1) are unbounded.

Condition 3.3. There exists $L>0$ and $\alpha \in(0,1]$ such that the following conditions are satisfied under Conditions 2.2 and 2.3 respectively:

$$
\begin{aligned}
(I): & 0<\sigma^{2}(t, x) \leq L\left(1+|\ln (\ln (x))| 1_{x>1}\right), \quad(t, x) \in \mathbf{R} \times \mathbf{R}, \\
& |r(t, x)| \leq L, \quad(t, x) \in \mathbf{R} \times \mathbf{R}, \\
(I I): & |\sigma(t, x)|+|r(t, x)| \leq \frac{L}{|x|^{\alpha}}+L, \quad x>0 .
\end{aligned}
$$

Lemma 3.4. Assume that Condition 3.3 is satisfied. Then, for all $p \geq 0$, there exists a constant $C_{p}$ independent of $\kappa$ such that $\mathbf{E}_{\sup _{u \leq T}}\left(S_{\kappa}(u)\right)^{p} \leq C_{p}$.

Lemma 3.5. Assume that Condition 3.3 is satisfied. Then, for all $p \geq 0$, there exists a constant $C$ such that $E \sup _{u \leq T}\left(S_{u}\right)^{p} \leq C_{p}$.

Lemma 3.6. Suppose that Condition 3.2 is satisfied for some $p>0$. Moreover, assume that Condition 3.3 (II) holds if $\sigma_{\kappa}=\sigma\left(\cdot, \cdot \vee \kappa^{-1}\right)$. Then, for all 
$l \geq 1, l \in \mathbb{N}$, there exists a constant $C_{l, p}$ such that

$$
\begin{aligned}
& \sigma_{\kappa}=\sigma(\cdot, \cdot \wedge \kappa) \Rightarrow \mathbf{E} \sup _{t \in[0, T]}\left|S_{\kappa}(t)-S_{t}\right|^{p} \leq \frac{C_{l, p}}{\kappa^{l}}, \\
& \sigma_{\kappa}=\sigma\left(\cdot, \cdot \vee \kappa^{-1}\right) \Rightarrow \mathbf{E} \sup _{t \in[0, \tau \wedge T]}\left|S_{\kappa}(t)-S_{t}\right|^{p} \leq C_{p} \alpha(\tau, \kappa),
\end{aligned}
$$

where $\alpha(\tau, \kappa) \rightarrow 0$, as $\kappa \rightarrow \infty$.

This result shows that $S$ may be approximated by $S_{\kappa}$ in the $L^{p}$ sense, $p>0$, as $\kappa \rightarrow \infty$.

\subsection{Picard iterations}

In this section, we construct iterated Doleans-Dade processes $S_{\kappa, m}, m \in \mathbf{N}$, such that $S_{\kappa, m}$ converge to $S_{\kappa}$ when $m \rightarrow \infty$. To do so, we introduce the following scheme:

$$
\begin{aligned}
S_{\kappa, 0} & :=S_{0}, \\
S_{\kappa, m+1}(t) & :=F_{t}\left(r_{\kappa, m+1}\right) \mathcal{E}_{t}\left(\sigma_{\kappa, m+1}\right), \quad t \in[0, T],
\end{aligned}
$$

where

$$
\begin{aligned}
\sigma_{\kappa, m+1}(t):=\sigma_{\kappa}\left(t, S_{\kappa, m}(t)\right), & m \geq 0, \quad t \in[0, T], \\
r_{\kappa, m+1}(t):=r_{\kappa}\left(t, S_{\kappa, m}(t)\right), & m \geq 0, \quad t \in[0, T] .
\end{aligned}
$$

Notice that we have

$$
S_{\kappa, m+1}(t)=S_{0} \exp \left[\int_{0}^{t} \sigma_{\kappa}\left(u, S_{\kappa, m}(u)\right) d W_{u}+\int_{0}^{t} \gamma_{\kappa}\left(u, S_{\kappa, m}(u)\right) d u\right] .
$$

Let us introduce

$$
Y_{\kappa, m}(t):=\log S_{\kappa, m}(t)-\log S_{0}, \quad t \in[0, T] .
$$

This process satisfies the following s.d.e.

$$
d Y_{\kappa, m+1}(t)=\sigma_{\kappa}\left(t, S_{\kappa, m}(t)\right) d W_{t}+\gamma_{\kappa}\left(t, S_{\kappa, m}(t)\right) d t
$$

We shall prove the convergence of $S_{\kappa, m}$ to $S_{\kappa}$ in $L^{2}$ as $m \rightarrow \infty$. 
Lemma 3.7. The sequence $Y_{\kappa, m}$ converges in $L^{4}$ to $Y_{\kappa, \infty}$ and

$$
\begin{aligned}
& \left\|\sup _{u \leq T}\left|Y_{\kappa, m+1}(u)-Y_{\kappa, m}(u)\right|\right\|_{2} \leq C\left(1+S_{0}\right)\left(C_{\kappa}\right)^{m+1} \frac{\sqrt{T}^{m+1}}{\sqrt{(m+1) !}} \\
& \left\|\sup _{u \leq T}\left|Y_{\kappa, m+1}(u)-Y_{\kappa, m}(u)\right|\right\|_{4} \leq C\left(1+S_{0}\right)\left(C_{\kappa}\right)^{m+1} \frac{\left(T^{1 / 4}\right)^{m+1}}{(m+1) !)^{1 / 4}}
\end{aligned}
$$

where $C$ is a constant which does not depend on $\kappa$ and $C_{\kappa}$ is a constant which depends on $\kappa$.

Corollary 3.8. There exists constants $C, C_{\kappa}$ independent of $m$ such that

$$
\begin{aligned}
& \mathbf{E} \sup _{u \leq t}\left|Y_{\kappa, \infty}(u)-Y_{\kappa, m}(u)\right|^{2} \leq C\left(1+S_{0}^{2}\right) \sum_{j=m+1}^{\infty} \frac{\left(C_{\kappa}\right)^{j}}{j !}, \\
& \mathbf{E} \sup _{u \leq t}\left|Y_{\kappa, \infty}(u)-Y_{\kappa, m}(u)\right|^{4} \leq C\left(1+S_{0}^{4}\right) \sum_{j=m+1}^{\infty} \frac{\left(C_{\kappa}\right)^{j}}{j !} .
\end{aligned}
$$

Lemma 3.9. For every $\kappa$, there exists constants $C, C_{\kappa}$ such that

$$
\begin{aligned}
\mathbf{E} \sup _{t \in[0, T]}\left(S_{\kappa, m+1}(t)-S_{\kappa, m}(t)\right)^{2} & \leq C_{\kappa} \sqrt{\mathbf{E} \sup _{t \in[0, T]}\left(Y_{\kappa, m}(t)-Y_{\kappa, m-1}(t)\right)^{4}} \\
& \leq C_{\kappa} \frac{\left(C_{\kappa}\right)^{m}}{\sqrt{m !}}
\end{aligned}
$$

By the lemma above, we deduce that $S_{\kappa, m}$ converges to $S_{\kappa, \infty}$ in $L^{2}$ with respect to the uniform convergence.

Lemma 3.10. For every $\kappa$, there exists a constant $C_{\kappa}$ such that

$$
\mathbf{E} \sup _{t \in[0, T]}\left(S_{\kappa, \infty}(t)-S_{\kappa, m}(t)\right)^{2} \leq C_{\kappa} \sum_{j=m}^{\infty} \frac{\left(C_{\kappa}\right)^{j}}{\sqrt{j !}} .
$$

Note that the limit processes satisfy

$$
\begin{aligned}
& d Y_{\kappa, \infty}(t)=\sigma_{\kappa}\left(t, S_{\kappa, \infty}(t)\right) d W_{t}+\gamma_{\kappa}\left(t, S_{\kappa, \infty}(t)\right) d t \\
& d S_{\kappa, \infty}(t)=S_{\kappa, \infty}(t) \sigma_{\kappa}\left(t, S_{\kappa, \infty}(t)\right) d W_{t}+S_{\kappa, \infty}(t) r_{\kappa}\left(t, S_{\kappa, \infty}(t)\right) d t
\end{aligned}
$$

It follows that $S_{\kappa, \infty}=S_{\kappa}$ and $Y_{\kappa, \infty}=Y_{\kappa}$ since uniqueness holds. 


\subsection{Volatility discretization based on Picard iterations.}

In the following, we suppose without loss of generality that $T / n \leq 1$ if $n$ is large enough. We recursively define the processes $S_{\kappa, m, n}, \sigma_{\kappa, m+1, n}, m \geq 0$, as follows:

$$
\begin{aligned}
S_{\kappa, 0, n} & :=S_{0}, \\
\sigma_{\kappa, 1, n}(t) & :=\sigma_{\kappa}\left(t_{i}, S_{0}\right), \quad r_{\kappa, 1, n}(t):=r_{\kappa}\left(t_{i}, S_{0}\right), \quad t_{i} \leq t<t_{i+1}, \\
S_{\kappa, m, n}(t) & :=F_{t}\left(r_{\kappa, m, n}\right) \mathcal{E}_{t}\left(\sigma_{\kappa, m, n}\right), \quad m \geq 1, \quad t \in[0, T], \\
\sigma_{\kappa, m+1, n}(t) & =\sigma_{\kappa}\left(t^{(n)}, S_{\kappa, m, n}^{(n)}(t)\right), \quad m \geq 1, \quad t \in[0, T], \\
r_{\kappa, m+1, n}(t) & =r_{\kappa}\left(t^{(n)}, S_{\kappa, m, n}^{(n)}(t)\right), \quad m \geq 1, \quad t \in[0, T] .
\end{aligned}
$$

where we use the notation $t^{(n)}=t_{i}$ if $t_{i} \leq t<t_{i+1}$ and we recall that

$$
\begin{aligned}
& \mathcal{E}_{t}\left(\sigma_{\kappa, m, n}\right)=\exp \left[\int_{0}^{t} \sigma_{\kappa}\left(u^{(n)}, S_{\kappa, m-1, n}^{(n)}(u)\right) d W_{u}-\frac{1}{2} \int_{0}^{t} \sigma_{\kappa}^{2}\left(u^{(n)}, S_{\kappa, m-1, n}^{(n)}(u)\right) d u\right] \\
& F_{t}\left(r_{\kappa, m, n}\right)=S_{0} \exp \left[\int_{0}^{t} r_{\kappa}\left(u^{(n)}, S_{\kappa, m-1, n}^{(n)}(u)\right) d u\right], \quad m \geq 1, \quad t \in[0, T] .
\end{aligned}
$$

We also define $Y_{\kappa, m, n}=\ln \left(S_{\kappa, m, n} / S_{0}\right)$.

Lemma 3.11. For all $\kappa>0$, there exists constants $\alpha_{\kappa}, C_{\kappa}>0$ such that for all $m, n \geq 1$ :

$$
\mathbf{E} \sup _{t \in[0, T]}\left|S_{\kappa, m}(t)-S_{\kappa, m, n}(t)\right|^{2} \leq \frac{\alpha_{\kappa} C_{\kappa}^{m}}{n}
$$

\subsection{Main result}

In the following, we follow the convention that $\tilde{\tau}^{\kappa, m, n}=+\infty$ if $P(\tau \leq T)=0$. Otherwise, $\tilde{\tau}^{\kappa, m, n}$ is defined as

$$
\tilde{\tau}^{\kappa, m, n}:=\inf \left\{t: S_{\kappa, m, n}^{(n)}(t) \leq \kappa^{-1}\right\}
$$

Theorem 3.12. Assume that Conditions 2.2 or 2.3 hold and suppose that $G$ is Lipschitz and consistent of order $n$ with respect to discretization. Suppose that $\tau=+\infty$ a.s. under Condition 2.2. Then, for $\kappa>0$ and $m, n \in \mathbb{N}$, there 
are some positive constants $\alpha_{\kappa}, C_{\kappa}$ and sequences $\varepsilon_{\kappa}>0, \varepsilon_{\kappa, m}>0$, and $\varepsilon_{\kappa, m, n}$ such that

$$
\left|\mathbf{E} G(S) 1_{\tau>T}-\mathbf{E} G\left(S_{\kappa, m, n}^{(n)}\right) 1_{\tilde{\tau}^{\kappa, m, n}>T}\right| \leq \varepsilon_{\kappa}+\varepsilon_{\kappa, m}+\frac{\alpha_{\kappa}\left(C_{\kappa}\right)^{m}}{n}+\varepsilon_{\kappa, m, n},
$$

where $\varepsilon_{\kappa} \rightarrow 0$ as $\kappa \rightarrow \infty, \varepsilon_{\kappa, m} \rightarrow 0$ as $m \rightarrow \infty, \varepsilon_{\kappa, m, n} \rightarrow 0$ as $n \rightarrow \infty$. Moreover, $\varepsilon_{\kappa, m, n}=0$ if $P(\tau \leq T)=0$.

Remark 3.13. In practice, we first fix $\kappa$ large enough and we fix $m$ large enough so that $\epsilon_{\kappa, m}$ is sufficiently small. At last, we choose $n$ large enough. The algorithm's complexity allowing to compute this scheme is then mn. Notice that in the case where $G(S)$ only depends on $S_{T}$ and $G(0)=0$, then $\mathbf{E} G(S)=\mathbf{E} G(S) 1_{\tau>T}$ which is approximated by $\mathbf{E} G\left(S_{\kappa, m, n}^{(n)}\right) 1_{\tilde{\tau}^{\kappa, m, n}>T}$.

\section{Numerical example: the CEV model}

The Constant Elasticity of Variance (CEV) model is defined by the dynamics

$$
d S_{t}=\sigma S_{t}^{\beta} d W_{t}, \quad t \in[0, T]
$$

where the parameter $\beta$ is called the elasticity. We suppose that $\sigma=\sigma_{L N} S_{0}^{1-\beta}$ where $\sigma_{L N}$ is the effective lognormal volatility. This implies that the volatility depends on $\beta$ as in [18], see also [5, 21] for more details on this model.

Consider the financial market model defined by a Bond $B \equiv 1$ and the risky asset price $S$. We recall that $S$ is a martingale if $\beta \leq 1$. If $\beta>1, S$ is a strict local martingale, i.e. is not a martingale. In both cases, there is no arbitrage opportunity when using predictable strategies generating portfolio processes which are bounded from below, see [4, Section 3.2]. Indeed, any self-financing portfolio process which is bounded from below is a supermartingale.

Let $h_{T}$ be a European or Asian contingent claim. Suppose that $h_{T}$ is bounded, which is the case for a Put option. Then, by the predictable representation theorem, the martingale $V_{t}=\mathbf{E}\left(h_{T} \mid \mathcal{F}_{t}\right), t \in[0, T]$, is a self-financing portfolio process such that $V_{T}=h_{T}$. We deduce that the unique price for the payoff $h_{T}$ is well defined as the expectation $V_{0}=\mathbf{E} h_{T}$. In the following, we illustrate 
our method by estimating $V_{0}=\mathbf{E} h_{T}$ when $\beta>1$ and $\beta<1$ respectively. The price for the Call option may be easily deduced from the Put-Call parity and the analytic expression of the expectations $\mathbf{E} S_{t}, t \in[0, T]$, see [18].

\subsection{The CEV model with elasticity $\beta>1$}

Let us introduce $\delta=(1-2 \beta)(1-\beta)^{-1}$. We suppose that $\delta>2$ or equivalently $\beta>1$ so that $S$ is a strictly positive process. The price of the Call option with strike $K>0$ is then given in [18] by

$p^{\text {Call }}\left(S_{0}, K, \sigma, \delta, T\right)=S_{0}\left(\Gamma\left(\frac{\delta}{2}-1, \frac{S_{0}}{2 T}\right)-\chi^{\prime 2}\left(\frac{S_{0}}{T}, \delta-2, \frac{\tilde{K}}{T}\right)\right)-K \chi^{\prime 2}\left(\frac{\tilde{K}}{T}, \delta, \frac{S_{0}}{T}\right)$

where $\tilde{K}:=\frac{K^{2(1-\alpha)}}{\sigma^{2}(1-\alpha)^{2}}, \Gamma$ is the normalised incomplete gamma function

$$
\Gamma(z, x)=\frac{1}{\Gamma(z)} \int_{0}^{x} t^{z-1} e^{-t} d t
$$

and $\chi^{\prime 2}(x, k, \lambda)$ is the cumulative density function of the non-central chisquared distribution with degrees of freedom $k$ and non-centrality parameter $\lambda$. The price of the Put option with strike $K$ is deduced as

$$
p^{\text {Put }}\left(S_{0}, K, \sigma, \delta, T\right)=p^{\text {Call }}\left(S_{0}, K, \sigma, \delta, T\right)-S_{0} \Gamma\left(1-\frac{\delta}{2}, \frac{S_{0}}{2 T}\right)+K .
$$

In [18], the price of the Put option is computed analytically for several values of $\beta>1$ when $T=1, S_{0}=100, \sigma_{L N}=20 \%$ and $K=90,100,110$. These analytical values are compared to the ones obtained by simulating the terminal value $X_{T}:=\frac{S_{T}^{2(1-\beta)}}{\sigma^{2}(1-\beta)^{2}}$. In the following, we compare these results to the values we obtain when using our method. Actually, we can not expect to obtain the convergence of $S_{\kappa}$ towards $S$ in the $L^{p}$ sense, $p \geq 1$, since Condition 3.2 does not a priori hold for $p \geq 1$, see also Condition 3.3 (I). Indeed, $\mathbf{E} S_{\kappa}(t)=S_{0}$ for all $t$ since $S_{\kappa}$ is a square integrable martingale. On the other hand, $\mathbf{E} S_{t}<S_{0}$ for all $t>0$ as shown in [18] since $S$ is a strict local martingale. Therefore, $\mathbf{E} S_{\kappa}(t)$ does not converge to $\mathbf{E} S_{t}$ as $\kappa \rightarrow \infty$. Nevertheless, when the payoff function $g \geq 0$ is bounded, the dominated convergence theorem yields that 
$g\left(S_{\kappa}\right) \rightarrow g(S)$ in the $L^{p}$ sense as $\kappa \rightarrow \infty, p \geq 1$, which is enough for our purpose. Precisely, since $S=S_{\kappa}$ on $\tau^{\kappa} \geq T$, we get that

$$
\mathbf{E}\left|g\left(S_{\kappa}\right)-g(S)\right|^{p} \leq(\max g)^{p} \mathrm{P}\left(\sup _{t \in[0, T]} S_{t} \geq \kappa\right) \rightarrow 0, \text { as } \kappa \rightarrow \infty .
$$

This implies that Theorem 3.12 still hold.

Let us compare the Picard method to the analytic one and to the backward Euler-Maruyama scheme (BEM), see [15]. In the following, we choose $m=3$ and $\sigma_{\kappa}(u, x):=\sigma(u, x \wedge \kappa)$ where

$$
\kappa=S_{0}\left(1+\frac{5}{1+\beta^{2}}\right) .
$$

This choice has been empirically chosen to improve the performance of our results for the Put option prices and then implemented for the Asian options. Recall that the volatility of the model also depends on $\beta$. If $\beta=1.5$, we observed that the approximation error is not smaller if we increase to much $\kappa$. The reason is very simple: choosing $\kappa$ very large is equivalent to suppose that $\kappa=+\infty$ as the maximum of $S$ over all simulated trajectories is finite since we fix a finite number of dates $n+1$ and a finite number of simulations $N$. Theoretically, if $\kappa$ is very large, we need to increase the number of dates $n$ as the Lipschitz constant is larger, which is more time consuming.

We choose $N=15000$ simulations and $n=1000$ so that the discretization step is $T / n$. We consider the case $K=100$. The presented results are averages we obtain by repeating more than 1000 times the same simulations so that the relative standard deviation of the results is smaller than $5 \%$ of the estimated averages.

Naturally, the computation time needed for the analytic method $(<0.01)$ is negligible contrary to the needed time for other methods which require to simulate all the trajectories.

In the following, we compare our method to the Monte Carlo one of [18] and the BEM method as well (see [15, Section 2, Section 3.4]), requiring that the radius of the $95 \%$ confidence interval is less than 0.025 when applying the law 


\begin{tabular}{|c|c|c|c|c|c|c|c|}
\hline$\beta$ & Picard & Time in s. & BEM & Time in s. & Analytic & Err. (P) & Err. (BEM) \\
\hline 1.5 & 7.8602 & 6.38 & 7.8839 & 0.78 & 7.9696 & $1.4 \%$ & $1.08 \%$ \\
2 & 7.8995 & 6.19 & 7.8930 & 0.76 & 7.9789 & $1.01 \%$ & $1.08 \%$ \\
2.5 & 7.9948 & 6.09 & 7.9087 & 0.79 & 7.9960 & $0.02 \%$ & $1.09 \%$ \\
3 & 8.1332 & 6.08 & 8.9329 & 0.76 & 8.0211 & $1.4 \%$ & $1.10 \%$ \\
3.5 & 8.2779 & 6.06 & 7.9686 & 0.80 & 8.0560 & $2.76 \%$ & $1.09 \%$ \\
4 & 8.4006 & 6.06 & 8.0200 & 0.81 & 8.1033 & $3.67 \%$ & $1.03 \%$ \\
4.5 & 8.4886 & 6.13 & 8.0841 & 0.80 & 8.1639 & $3.98 \%$ & $0.98 \%$ \\
5 & 8.5348 & 6.08 & 8.1583 & 0.78 & 8.2345 & $3.65 \%$ & $0.93 \%$ \\
5.5 & 8.5344 & 5.936 & 8.2365 & 0.78 & 8.3084 & $2.72 \%$ & $0.87 \%$ \\
6 & 8.5015 & 5.906 & 8.3086 & 0.80 & 8.3786 & $1.47 \%$ & $0.84 \%$ \\
6.5 & 8.4499 & 6.096 & 8.3715 & 0.78 & 8.4396 & $0.13 \%$ & $0.81 \%$ \\
7 & 8.3821 & 6.401 & 8.4228 & 0.82 & 8.4882 & $1.26 \%$ & $0.77 \%$ \\
\hline
\end{tabular}

TABLE 1. Put option prices using Picard iterations and the analytic expression respectively for different values of $\beta>1$.

\begin{tabular}{|c|c|c|c|c|c|c|c|}
\hline$\beta$ & Picard & Comput. Time & BEM & Time & MC [32] & Time & Error \\
\hline 1.5 & 7.961482891 & 268.748 & 7.9572 & 31.73 & 7.958596698 & 20.756 & $0.03 \%$ \\
2 & 8.001945176 & 276.356 & 7.9692 & 28.28 & 7.968447144 & 12.571 & $0.42 \%$ \\
2.5 & 8.096442435 & 286.754 & 7.9841 & 26.05 & 7.981469166 & 13.68 & $1.44 \%$ \\
3 & 8.231139717 & 298.65 & 8.0099 & 23.60 & 8.002688168 & 13.098 & $2.86 \%$ \\
3.5 & 8.382673524 & 317.32 & 8.0483 & 22.09 & 8.050143346 & 10.78 & $4.14 \%$ \\
4 & 8.507583601 & 338.079 & 8.0948 & 20.57 & 8.091346416 & 7.908 & $5.15 \%$ \\
4.5 & 8.594539064 & 345.449 & 8.1543 & 19.06 & 8.164748506 & 5.855 & $5.17 \%$ \\
5 & 8.631951141 & 351.436 & 8.2231 & 17.13 & 8.234170007 & 3.698 & $4.84 \%$ \\
\hline
\end{tabular}

TABLE 2. Put option prices using Picard iterations, BEM and Monte Carlo methods respectively for different values of $\beta>1$ so that the radius of the $95 \%$ confidence interval is less than 0.025 .

of large number. The simple Monte Carlo simulation is less time consuming. Nevertheless, we shall see that the two other methods outperform the Monte Carlo simulations as soon as we consider an Asian Put option with several dates.

It is also natural to compare our method to a simple Doléans-Dade scheme deduced from the dynamics (4.10) where the volatility function $\sigma(x)=\sigma x^{\beta-1}$ is replaced by $\sigma_{\kappa}(x)=\sigma(x \wedge \kappa)^{\beta-1}$. Inspired by the Doleans-Dade formula, we consider the Doléans-Dade scheme

$S_{t_{i+1}}^{n}=S_{t_{i}}^{n} \exp \left\{\sigma_{\kappa}\left(S_{t_{i}}^{n}\right) \Delta W_{t_{i+1}}-\frac{\sigma_{\kappa}^{2}\left(S_{t_{i}}^{n}\right)}{2} \Delta t_{i+1}\right\}, S_{0}^{n}=S_{0}, \quad i=0, \cdots, n-1$.

In the following table, we compare the approximate prices to the analytic ones for several values of $\beta$ by implementing the scheme until the error is smaller than the one observed in Table 1 by increasing $N$ with the bound $N \leq 5.10^{6}$. We choose $n=1000$ so that the time step is $T / n$. The strike is $K=100$ and $\kappa=400$. The error is still computed as the ratio between the difference of the two prices and the smallest one. 


\begin{tabular}{|c|c|c|c|c|}
\hline$\beta$ & Price (truncated Doléans-Dade scheme) & Comput. time in seconds & $N$ & Error \\
\hline 1.5 & 7.1412 & 535 & $5.10^{6}$ & $11.6 \%$ \\
2 & 7.1978 & 555 & $5.10^{6}$ & $10.85 \%$ \\
2.5 & 7.2544 & 553 & $5.10^{6}$ & $10.22 \%$ \\
3 & 7.3134 & 549 & $5.10^{6}$ & $9.68 \%$ \\
3.5 & 7.3779 & 556 & $5.10^{6}$ & $9.19 \%$ \\
4 & 7.4524 & 564 & $5.10^{6}$ & $8.73 \%$ \\
4.5 & 7.5999 & 541 & $5.10^{6}$ & $7.42 \%$ \\
5 & 8.0123 & 0.014 & 136 & $3.65 \%$ \\
5.5 & 8.4954 & 0.013 & 136 & $2.72 \%$ \\
6 & 10.7683 & 523 & $5.10^{6}$ & $28.5 \%$ \\
6.5 & 11.934 & 518 & $5.10^{6}$ & $41.4 \%$ \\
7 & 12.9042 & 518 & $5.10^{6}$ & $52 \%$ \\
\hline
\end{tabular}

TABLE 3. Put option prices with truncated Doléans-Dade scheme for different values of $\beta$.

We observe that the results are similar for smaller $\kappa=120$ as the errors are around $10 \%$ and computation times are about 520 seconds. If $\kappa=800$, we get similar results. The performance is still good for $\beta=5$ but also $\beta=4$ while it is bad for $\beta=5.5$ contrary to the case $\kappa=400$. The best results we can get seem to be when $\kappa=+\infty$, i.e. without any truncation, but remains bad. With a larger $n=10000$ and $N=15000$, errors go from $2.5 \%$ to $10.24 \%$ but computational times are larger than 14 seconds. With only $n=1000$, computation times are smaller, precisely about 1.5 seconds, but the errors are similar i.e. from $2 \%$ to $20 \%$. So, seemingly, it is more efficient to increase $N$ rather than $n$ if we aim to decrease the error. In any case, comparing with the results of Table 1, it is clear that our methods outperforms the simple truncated Euler scheme.

As to a direct discretization from (4.10), i.e. $S_{t_{i+1}}^{n}=S_{t_{i}}^{n}+\Sigma\left(S_{t_{i}}^{n}\right) \Delta W_{t_{i+1}}$, the Euler scheme with $n=1000$ and $N=15000$ explodes without truncation for $\beta \geq 3$ as we may expect. For $\beta \in\{1.5,2,2.5\}$, the scheme appears to be efficient as the error is small $(\leq 1.4 \%)$ and the computation time is also smaller $(\simeq 1.6$ seconds $)$. With the truncation $\Sigma(x)=\sigma(x \wedge \kappa)^{\beta}$ where $\kappa=300$, the errors are about $1.5 \%$ for the first values of $\beta>1$ while the errors go to $4.6 \%$ for $\beta=5$ to $769 \%$ for $\beta=7$. Therefore, a truncation from the simple Euler scheme seems to be conceivable only for values of $\beta$ smaller than 5 .

At last, we shall see that our method outperforms the classical one based on Monte Carlo simulations as soon as we consider an Asian Put option of the form $\left(K-\left(S_{T_{1}}-\cdots S_{T_{J}}\right) / J\right)^{+}$where $J \geq 3$ and $T_{1}<T_{2}<\cdots<T_{J}=T$. 
Let us first consider the case $J=2$. In that case, by conditioning, we rewrite $\mathbf{E}\left(2 K-S_{T_{1}}-S_{T_{2}}\right)^{+}=\mathbf{E} P\left(S_{T_{1}}\right)$ where, by the Markov property, $P(s)$ is the price of the Put option of strike $2 K-s$ in the CEV model with initial value $s$ and horizon date $T_{2}-T_{1}$. As shown in [18], it is possible to directly simulate the distribution of $S_{T_{1}}$. We then analytically deduce the price $P\left(S_{T_{1}}\right)$ for each simulated value of $S_{T_{1}}$. By the law of large numbers, we then deduce an approximation of $\mathbf{E} P\left(S_{T_{1}}\right)$. With the same parameters as before, we obtain the following results when $T_{1}=T / 2$ and $T_{2}=T=1$.

\begin{tabular}{|c|c|c|c|c|c|c|c|c|c|c|}
\hline$\beta$ & P & Time & BEM (B) & Time & MC & Time & Error P-C & Euler (E) & Error E-C & Error B-C \\
\hline 1.5 & 6.2284 & 6.02 & 6.3342 & 0.52 & 6.3147 & 0.328 & $1.39 \%$ & 6.3983 & $1.32 \%$ & $0.31 \%$ \\
2 & 6.2549 & 6.4 & 6.3379 & 0.52 & 6.3198 & 0.133 & $1.04 \%$ & 6.4052 & $1.35 \%$ & $0.29 \%$ \\
2.5 & 6.3142 & 6.178 & 6.3466 & 0.53 & 6.3297 & 0.121 & $0.25 \%$ & 6.4165 & $1.37 \%$ & $0.27 \%$ \\
3 & 6.4027 & 6.159 & 6.3603 & 0.52 & 6.3438 & 0.113 & $0.93 \%$ & 6.4321 & $1.39 \%$ & $0.26 \%$ \\
3.5 & 6.5011 & 6.398 & 6.3780 & 0.53 & 6.3625 & 0.118 & $2.18 \%$ & 6.4520 & $1.41 \%$ & $0.24 \%$ \\
4 & 6.5950 & 6.235 & 6.3998 & 0.53 & 6.3864 & 0.101 & $3.27 \%$ & 6.4772 & $1.42 \%$ & $0.21 \%$ \\
4.5 & 6.6737 & 6.25 & 6.4269 & 0.53 & 6.4166 & 0.098 & $4 \%$ & 6.5162 & $1.55 \%$ & $0.16 \%$ \\
5 & 6.7267 & 6.188 & 6.4623 & 0.52 & 6.4544 & 0.101 & $4.22 \%$ & 6.7340 & $4.33 \%$ & $0.12 \%$ \\
5.5 & 6.7465 & 5.967 & 6.5060 & 0.53 & 6.5003 & 0.102 & $3.79 \%$ & 7.5778 & $16.58 \%$ & $0.09 \%$ \\
6 & 6.7411 & 5.968 & 6.5571 & 0.53 & 6.5530 & 0.102 & $2.87 \%$ & 12.7244 & $94.18 \%$ & $0.06 \%$ \\
6.5 & 6.7192 & 5.997 & 6.6099 & 0.53 & 6.6102 & 0.100 & $1.65 \%$ & 25.4794 & $285.46 \%$ & $0.00 \%$ \\
7 & 6.6820 & 6.000 & 6.6638 & 0.53 & 6.6687 & 0.100 & $0.20 \%$ & 51.2649 & 668.74 & $0.07 \%$ \\
\hline
\end{tabular}

TABLE 4. Asian Put option prices with two dates using Picard iterations (P), the Monte Carlo methods combined with the analytic expression (MC) and a truncated Euler scheme (E) respectively. The errors are computed between the Picard method (P) and the Euler scheme (E) respectively with respect to the combined method $(C)$.

\begin{tabular}{|c|c|c|c|c|c|c|c|c|}
\hline$\beta$ & P & Time in s. & BEM (B) & Time & MC & Time & Error P-C & Error B-C \\
\hline 1.5 & 6.293809919 & 171.191 & 6.2947 & 20.49 & 6.307557882 & 10.375 & $0.22 \%$ & $0.20 \%$ \\
2 & 6.314878756 & 173.881 & 6.3012 & 18.93 & 6.311235573 & 3.961 & $0.06 \%$ & $0.16 \%$ \\
2.5 & 6.373160059 & 179.582 & 6.3126 & 17.68 & 6.320525289 & 3.429 & $0.84 \%$ & $0.12 \%$ \\
3 & 6.460785842 & 186.615 & 6.3216 & 16.33 & 6.331600424 & 3.015 & $2.04 \%$ & $0.16 \%$ \\
3.5 & 6.559108888 & 198.54 & 6.3398 & 15.48 & 6.347881289 & 2.524 & $3.33 \%$ & $0.13 \%$ \\
4 & 6.655375191 & 205.6 & 6.3670 & 14.41 & 6.366022368 & 2.317 & $4.55 \%$ & $-0.02 \%$ \\
4.5 & 6.734410841 & 216.076 & 6.3994 & 13.56 & 6.390785176 & 2.13 & $5.38 \%$ & $-0.13 \%$ \\
5 & 6.781869529 & 219.877 & 6.4358 & 12.62 & 6.42642745 & 2.009 & $5.54 \%$ & $-0.15 \%$ \\
\hline
\end{tabular}

TABle 5. Asian Put option prices with two dates using Picard iterations and the Monte Carlo methods combined with the analytic expression respectively such that the $95 \%$ confidence interval radius is smaller than 0.025 .

As we may observe, it is still advantageous to combine the Monte Carlo method with the analytic one in the case of an Asian option with only two dates. Moreover, we still observe that the simple truncated Euler scheme is efficient for $\beta \leq 5$ while it produces a large convergence error if $\beta>5$ contrary to the Picard iterations.

Let us now suppose that $J=3$. By the Markov property, we rewrite after conditioning $\mathbf{E}\left(3 K-S_{T_{1}}-S_{T_{2}}-S_{T_{3}}\right)^{+}=\mathbf{E} P\left(S_{T_{1}}\right)$ where $P(s)$ is the price 
of the Asian Put option of strike $3 K-s$ in the CEV model with initial value $s$, intermediate date $T_{2}-T_{1}$ and horizon date $T_{3}-T_{1}$. By the Monte Carlo method, we simulate $S_{T_{1}}$ so that we deduce $P\left(S_{T_{1}}\right)$ using the pricer for two dates we have introduced before. In that case, the complexity explodes as we need to run two successive Monte Carlo schemes. With the same parameters but only with $\beta=1.5$ or $\beta=7$ and $T_{1}=T / 3, T_{2}=2 T / 3$ and $T_{3}=T=1$, we get the following results:

\begin{tabular}{|c|c|c|c|c|c|c|c|c|c|c|}
\hline$\beta$ & P & Time & BEM & Time & MC & Time & Error P-C & Euler (E) & Error E-C & Error B-C \\
\hline 1.5 & 5.3788 & 61.73 & 5.6768 & 7.87 & 5.2698 & 8333 & $2.07 \%$ & 5.8172 & $10.39 \%$ & $7.72 \%$ \\
7 & 5.6278 & 61.83 & 5.9535 & 8.36 & 5.5188 & 2306 & $1.98 \%$ & 10.2290 & $85.35 \%$ & $7.88 \%$ \\
\hline
\end{tabular}

TABle 6. Asian Put option price with three dates when $\beta=1.5$ or $\beta=7$ using Picard iterations, the Monte Carlo methods combined with the analytic expression and the truncated Euler scheme respectively. The errors are computed between the Picard method $(P)$ and the Euler scheme (E) respectively with respect to the combined method $(C)$.

\begin{tabular}{|c|c|c|c|c|c|c|c|c|c|}
\hline$\beta$ & P & Time & BEM & Time & CI & MC & Time & Error P-C & Error B-C \\
\hline 1.5 & 5.345 & 1322 & 5.7254 & 17.724 & 0.05 & 5.26 & 372089 & $1.62 \%$ & $8.85 \%$ \\
\hline
\end{tabular}

TABle 7. Asian Put option price with three dates using Picard iterations and the Monte Carlo methods combined with the analytic expression respectively such that the $95 \%$ confidence interval radius is smaller than 0.05 .

Conclusion: our method appears to outperform the technique based on the Monte Carlo methods combined with the analytic expression for Asian options with more than three dates as well as the truncated Euler scheme. Notice that computational times may be shortened in the Picard scheme by replacing the time-consuming computation of the exponential function by the first terms of its Taylor expansion. Nevertheless, the BEM method appears to be the most efficient even if, with three dates, the error in Table 7 is smaller for the Picard method. In the case where $\beta=2$, the simple truncated Euler scheme seems to be efficient provided that $\beta \leq 5$ as convergence errors and computation times are small. In the following section, we implement the CEV model for $\beta<1$. In that case, the BEM method does not work well. 


\subsection{The CEV model with elasticity $\beta \in(0,1)$}

Recall that Condition 2.3 holds when $\beta \in(0,1)$. Nevertheless, the required property that there exists an absorbing point only holds when $\beta \geq 0.5$ [18]. In this section, the parameters are $S_{0}=100, \sigma_{L N}=0.5, T=4, K=100$, $N=15000, n=20$ and $m=2$. The parameter $\kappa$ is empirically fixed to $\kappa=S_{0}\left(0.78-0.4 * \beta^{2}\right)$ for the Picard scheme and $\kappa=300$ for the truncated Euler scheme.

When implementing the BEM method, the implicit scheme requires to solve an equation of the form $g(X)=0$, e.g. using the Newton method. To do so, we fix an interval $[a, b]$ we expect the solution $X^{*}$ to belong to. Unfortunately, the algorithm stops as soon as $a$ is too large or $b$ too small as $[a, b]$ does not contain $X^{*}$. We observe this phenomenon even with $a=10^{-8}$ and $b=10^{8}$ so that we can not obtain any result.

We consider the Put option.

The methodology we follow consists in increasing the number of simulations $N$ until the estimated price belongs to the interval of radius $1 \%$ and centred at the analytic expression of the price. Otherwise, we stop $N$ to $2.10^{5}$. Repeating 1000 times the procedure, we deduce an average of the estimated price for each method and the needed time in milliseconds as well.

\begin{tabular}{|c|c|c|c|c|c|c|}
\hline$\beta$ & Truncated Euler scheme & Time $(\mathrm{ms})$ & Analytic & Time $(\mathrm{ms})$ & Error & \# of simulations \\
\hline 0 & 37.5155 & 18.254 & 39.0452 & $<0.01$ & $3.92 \%$ & 8618 \\
0.1 & 38.8543 & 4.662 & 39.0089 & $<0.01$ & $0.40 \%$ & 1891 \\
0.2 & 38.9200 & 1.806 & 38.9307 & $<0.01$ & $0.03 \%$ & 745 \\
0.3 & 38.8460 & 1.106 & 38.8210 & $<0.01$ & $0.06 \%$ & 460 \\
0.4 & 38.7139 & 1.164 & 38.6962 & $<0.01$ & $0.05 \%$ & 487 \\
0.5 & 38.5853 & 1.099 & 38.5753 & $<0.01$ & $0.03 \%$ & 456 \\
0.6 & 38.4624 & 2.287 & 38.4724 & $<0.01$ & $0.03 \%$ & 930 \\
0.7 & 38.2547 & 4.537 & 38.3928 & $<0.01$ & $0.36 \%$ & 1866 \\
0.8 & 37.4803 & 12.511 & 38.3368 & $<0.01$ & $2.23 \%$ & 5198 \\
0.9 & 36.9813 & 17.533 & 38.3041 & $<0.01$ & $3.45 \%$ & 7479 \\
\hline
\end{tabular}

TABLE 8. Put option prices using truncated Euler Scheme and the analytic expression respectively for different values of $\beta<1$.

Let us now present the approximated prices obtained by the Picard method with $n=20$. 


\begin{tabular}{|c|c|c|c|c|c|}
\hline$\beta$ & Picard & Comp. Time (ms) & Analytic & Error & \# of simulations \\
\hline 0 & 39.0421 & 1.299 & 39.0451 & $0.01 \%$ & 218 \\
0.1 & 38.9987 & 1.608 & 39.0088 & $0.03 \%$ & 269 \\
0.2 & 38.9235 & 1.759 & 38.9306 & $0.02 \%$ & 292 \\
0.3 & 38.8090 & 1.331 & 38.8209 & $0.03 \%$ & 219 \\
0.4 & 38.7007 & 1.477 & 38.6961 & $0.01 \%$ & 239 \\
0.5 & 38.5871 & 2.22 & 38.5753 & $0.03 \%$ & 367 \\
0.6 & 38.5036 & 3.112 & 38.4724 & $0.08 \%$ & 520 \\
0.7 & 38.4055 & 2.136 & 38.3928 & $0.03 \%$ & 357 \\
0.8 & 38.3374 & 1.336 & 38.3368 & $0.00 \%$ & 216 \\
0.9 & 38.1972 & 6.028 & 38.3041 & $0.28 \%$ & 991 \\
\hline
\end{tabular}

TABLE 9. Put option prices using Picard iterations and the analytic expression respectively for different values of $\beta<1$.

As we may observe, the Picard method outperforms the truncated euler method, i.e. provides smaller error with less computation time. For two dates Asian options we choose $n=40$ in order to work with the same mesh.

\begin{tabular}{|c|c|c|c|c|c|c|c|c|}
\hline$\beta$ & $(\mathrm{P})$ & Time $(\mathrm{ms})$ & MC $(\mathrm{C})$ & Time $(\mathrm{ms})$ & Error P-C & Euler $(\mathrm{E})$ & Time (ms) & Error E-C \\
\hline 0,5 & 28,8773 & 266,07 & 32,5530 & 0,103 & $12,73 \%$ & 28,0664 & 171,11 & $15,99 \%$ \\
0,6 & 31,3302 & 11,391 & 31,3744 & 0,124 & $0,14 \%$ & 30,9665 & 18,045 & $1,32 \%$ \\
0,7 & 30,7832 & 5,097 & 30,7640 & 0,137 & $0,06 \%$ & 30,7794 & 2,305 & $0,05 \%$ \\
0,8 & 30,6667 & 2,84 & 30,6624 & 0,149 & $0,01 \%$ & 30,6813 & 3,402 & $0,06 \%$ \\
0,9 & 30,6594 & 4,208 & 30,6532 & 0,194 & $0,02 \%$ & 30,6334 & 3,826 & $0,06 \%$ \\
\hline
\end{tabular}

TABLE 10. Asian Put option prices with two dates using Picard iterations (P), truncated Euler scheme (E) and the Monte Carlo methods combined with the analytic expression $(C)$. The errors are computed between the Picard method $(P)$ and the Euler scheme (E) respectively with respect to the combined method $(C)$.

Clearly, the combined method derived from [18] remains the most performant for two dates. Notice that the Picard method outperforms the truncated one. At last, with the combined method, it takes several hours to estimate the price of an Asian option with three dates $T i / 3, i=1,2,3$ and $T=4$. It only takes 78 seconds for the Picard method and the estimated price is 27.8456.

Conclusion: in the case where $\beta \in[0,1)$, the Picard method seems to be the best method comparatively to Euler truncated scheme, the BEM method or the combined method based on Monte Carlo simulations of the terminal price for more than three dates as the computation time is smaller and the errors are satisfactory. Otherwise, for one or two dates, the combined method derived from [18] appears to be efficient. 


\section{The Aï-Sahalia model}

The Aït-Sahalia model (see [15, Section 3.6]) satisfies the s.d.e.

$$
d S_{t}=\sigma S_{t}^{\rho} d W_{t}+\left(\alpha_{-1} S_{t}^{-1}-\alpha_{0}+\alpha_{1} S_{t}-\alpha_{t} S_{t}^{r}\right) d t
$$

where $\rho, r>1, \alpha_{i} \geq 0, i=-1, \cdots, 2$ and $\sigma>0$. The initial value is $S_{0}=0.2$ and the other parameters are $T=1, K=0$ for the Call option so that we shall estimate $E\left(S_{T}\right)$.

We choose $\alpha_{-1}=0.1$ and $\alpha_{i}=0$, if $i=0, \cdots, 2$. We fix $\sigma=0.2$ and $r=\rho=2$. The BEM method provides $E\left(S_{T}\right) \simeq 0.4906674$ in 6.689 m.s. The simple Euler scheme compute the value $E\left(S_{T}\right) \simeq 0.49050227$ in 1.227 m.s. while the Picard method estimates $E\left(S_{T}\right) \simeq 0.4905902$ in 19.517 m.s. for $m=10$. With $m=5$, it takes 9.832 m.s. but we get $E\left(S_{T}\right) \simeq 0.5041992$. If we choose the parameters $\alpha_{-1}=0.1, \alpha_{0}=1, \alpha_{1}=5$ and $\alpha_{2}=2$, by the BEM method, we get $E\left(S_{T}\right) \simeq 1.59841981$ in 7.531 m.s. while the simple Euler scheme provides $E\left(S_{T}\right) \simeq 1.6022864$ in 1.245 m.s. At last, it takes 18.747 m.s. for the Picard method to estimate $E\left(S_{T}\right) \simeq 1.6042199$ with $m=10$. On these examples, we see that the simple Euler scheme provides a rather accurate estimation of $E S_{T}$ in comparaison to the evaluation given by the BEM method that requires more time. Clearly, the Picard method is not efficient on this model in the sense that the needed time to obtain a small error is significantly larger.

\section{Example of mean reverting SDEs}

Let us consider the dynamics $d S_{t}=\left(1-S_{t}\right) d t+\gamma S_{t}^{\beta} d W_{t}$, where $W$ is a standard Brownian motion and $\beta \in[0.5,1)$. Here we choose $\beta=1$. Using the Lamperti transformation, we simulate the process $X_{t}=S_{t}^{-\beta+1}$ when implementing the BEM method. We noticed that the BEM scheme explodes because of the inversion problem that needs to be solved and the simple Euler scheme explodes in particular when $\beta$ is closed to 0.5. Otherwise, an improved convergent Milstein scheme is proposed by [6]. As mentioned in [6], 
the discretization step needs to be smaller than $\gamma^{-2}$ for the approximation process to be positive. This is indeed what we observed so that the needed time to compute appears to be significantly large for $\gamma$ large enough. In that case, the Picard method appears to be more efficient. Here we compare the two methods with $\gamma=1$ so that it is possible to get accurate estimations with the improved Milstein scheme in a reasonable computation time. The goal here is to present the accuracy of the Picard method. With the discrete dates $t_{k}^{n}=(T / n) k, k=0, \cdots, n$, the improved Milstein is given by:

$$
\begin{aligned}
S_{t_{k+1}^{n}}^{n}= & S_{t_{k}^{n}}^{n}+\left(1-S_{t_{k+1}^{n}}^{n}\right) \Delta t_{k+1}^{n}+\left[S_{t_{k}^{n}}^{n}\right]^{\beta} \Delta W_{t_{k}^{n}} \\
& +\frac{1}{2}(\beta-1)\left[S_{t_{k}^{n}}^{n}\right]^{2 \beta-1}\left(\left(\Delta W_{t_{k}}\right)^{2}-\Delta t_{k}\right) .
\end{aligned}
$$

We propose to compare the results obtained by implementing the Picard method without truncature, i.e. $\kappa=+\infty$, to the Milstein scheme as a reference. To do so, we first implement the Milstein scheme. We then compute the averaged needed time over 1000 experiments for the Picard method to provide an approximation at a relative distance from the Milstein estimation less than $1 \%$. As shown in the following table, the estimations are very accurate.

\begin{tabular}{|c|c|c|c|c|}
\hline$\beta$ & Milstein & Picard & Error & Time $(\mathrm{s})$ \\
\hline 0.5 & 0.9968 & 0.9980 & $0.12 \%$ & 1.103 \\
0.55 & 0.9969 & 0.9974 & $0.05 \%$ & 0.631 \\
0.6 & 0.9969 & 0.9977 & $0.07 \%$ & 0.633 \\
0.65 & 0.9969 & 0.9976 & $0.07 \%$ & 0.643 \\
0.7 & 0.9968 & 0.9977 & $0.09 \%$ & 0.656 \\
0.75 & 0.9967 & 0.9979 & $0.12 \%$ & 0.760 \\
0.8 & 0.9965 & 0.9972 & $0.07 \%$ & 0.835 \\
0.85 & 0.9961 & 0.9974 & $0.13 \%$ & 0.886 \\
0.9 & 0.9955 & 0.9965 & $0.10 \%$ & 0.979 \\
0.95 & 0.9946 & 0.9960 & $0.14 \%$ & 1.129 \\
1 & 0.9932 & 0.9945 & $0.12 \%$ & 0.853 \\
\hline
\end{tabular}

TABLE 11. Estimation of $E S_{T}$ with $T=6$ and $S_{0}=1$ for several values of $\beta$. 


\section{Conclusion}

The conclusion of this work is that it is not possible to say in general what the 'best' simulation technique is. Through the examples we present, the same technique may appear as very efficient or, on the contrary, inappropriate. The Picard technique seems to be appropriate for models where the diffusion coefficient is sublinear, e.g. for asian option pricing, while the BEM method is efficient when the diffusion coefficient is super linearly growing.

\section{Proofs}

Throughout the paper, we shall use the following convention: from a line to the next one, constants $K, \kappa$ or $C$ may designate different constants which are independent of any other current variables except maybe the fixed parameters of the model as the maturity date $T$ or Lipschitz constants. In the contrary case, we shall use notations like $C_{m}$ to stress the dependence on some parameter $m$.

Proof of Lemma 2.6. Let us denote $S=S_{r, \sigma}=F(r) \mathcal{E}(\sigma)$. Using the integration by part formula, we obtain that

$$
S_{t} t=\int_{0}^{t} S_{u} d u+\int_{0}^{t} u d S_{u}, \quad t \in[0, T] .
$$

On the other hand, a direct computation shows that

$$
\int_{0}^{T} S_{u}^{(n)} d u=-\int_{0}^{T} u d S_{u}^{(n)}+T S_{T}^{(n)} .
$$

Since $S_{T}^{(n)}=S_{T}$, we deduce that

$$
\int_{0}^{T} S_{u} d u-\int_{0}^{T} S_{u}^{(n)} d u=\int_{0}^{T} u d\left(S_{u}^{(n)}-S_{u}\right) .
$$


Observe that

$$
\begin{aligned}
\int_{0}^{T} u d S_{u}^{(n)} & =\sum_{i=1}^{n} t_{i}^{n} \Delta S_{t_{i}^{n}} \\
& =\sum_{i=1}^{n} t_{i}^{n} \int_{t_{i-1}^{n}}^{t_{i}^{n}} \sigma\left(u, S_{u}\right) S_{u} d W_{u}+\sum_{i=1}^{n} t_{i}^{n} \int_{t_{i-1}^{n}}^{t_{i}^{n}} r\left(u, S_{u}\right) S_{u} d u \\
& =\int_{0}^{T} I^{(n)}(u) \sigma\left(u, S_{u}\right) S_{u} d W_{u}+\int_{0}^{T} I^{(n)}(u) r\left(u, S_{u}\right) S_{u} d u
\end{aligned}
$$

where $I^{(n)}(u)=t_{i}$ if $u \in\left[t_{i-1}, t_{i}\right)$. It follows that

$$
\int_{0}^{T} S_{u} d u-\int_{0}^{T} S_{u}^{(n)} d u=\int_{0}^{T}\left(I^{(n)}(u)-u\right) \sigma\left(u, S_{u}\right) S_{u} d W_{u}+\int_{0}^{T}\left(I^{(n)}(u)-u\right) r\left(u, S_{u}\right) S_{u} d u
$$

As $\left|u^{(n)}(u)-u\right| \leq T / n$ and $r$ and $\sigma$ are supposed to be Lipschitz, we deduce by the Cauchy-Schwarz inequality and the Ito isometry the following sequence of inequalities:

$$
\begin{aligned}
\mathbf{E} \mid G(S))-G\left(S^{(n)}\right) \mid \leq & C_{T} \mathbf{E}\left|\int_{0}^{T}\left(I^{(n)}(u)-u\right) \sigma\left(u, S_{u}\right) S_{u} d W_{u}\right| \\
& +C_{T} \mathbf{E}\left|\int_{0}^{T}\left(I^{(n)}(u)-u\right) r\left(u, S_{u}\right) S_{u} d u\right| \\
\mathbf{E}\left|G(S)-G\left(S^{(n)}\right)\right| \leq & C_{T} \sqrt{C(\sigma) \int_{0}^{T}\left(I^{(n)}(u)-u\right)^{2} \mathbf{E}\left(1+S_{u}^{4}\right) d u} \\
& +C_{T}(r) \int_{0}^{T}\left|I^{(n)}(u)-u\right| \mathbf{E}\left(1+S_{u}^{2}\right) d u \\
\leq & \frac{C_{G} \sqrt{C(\sigma)}}{n} \sqrt{\int_{0}^{T}\left(1+\mathbf{E} S_{u}^{4}\right) d u} \\
& +\frac{C_{T}(r)}{n} \int_{0}^{T} \mathbf{E}\left(1+S_{u}^{2}\right) d u, \\
& \frac{C_{G} \sqrt{C(\sigma)}}{n} \sqrt{\int_{0}^{T}\left(1+\mathbf{E} S_{u}^{4}\right) d u} \\
& +\frac{C_{T}(r)}{n} \sqrt{\int_{0}^{T} \mathbf{E}\left(1+S_{u}^{2}\right)^{2} d u .}
\end{aligned}
$$

The conclusion follows. 
Lemma 8.1. Suppose that Condition 2.3 holds. Let $\kappa>0$. Then, there exists a constant $C_{\kappa}$ depending on $\kappa$ such that for all $x, y>0$ and $t \in \mathbf{R}_{+}$,

$$
\left|\sigma_{\kappa}(t, x)-\sigma_{\kappa}(t, y)\right| \leq C_{\kappa} \sqrt{|x-y|}
$$

Proof. Under Conditions 2.3, we obtain the following chain of inequalities

$$
\begin{aligned}
\left|\sigma_{\kappa}(t, x)-\sigma_{\kappa}(t, y)\right| & =\left|\sigma_{\kappa}\left(t, x \vee \kappa^{-1}\right)-\sigma_{\kappa}\left(t, y \vee \kappa^{-1}\right)\right|, \\
& \leq C_{\kappa}\left|\ln \left(x \vee \kappa^{-1}\right)-\ln \left(y \vee \kappa^{-1}\right)\right| \\
& \leq C_{\kappa}\left|\ln (x) \vee \ln \left(\kappa^{-1}\right)-\ln (y) \vee \ln \left(\kappa^{-1}\right)\right| \\
& \leq 2 C_{\kappa}\left|\ln (\sqrt{x}) \vee \ln \left(\kappa^{-1 / 2}\right)-\ln (\sqrt{y}) \vee \ln \left(\kappa^{-1 / 2}\right)\right|, \\
& \leq C_{\kappa}|\sqrt{x}-\sqrt{y}| \leq C_{\kappa} \sqrt{|x-y|}
\end{aligned}
$$

The last inequalities are due to the fact that the mapping $z \mapsto \ln (z) \vee$ $\ln \left(\kappa^{-1 / 2}\right)$ is Lipschitz and the square root function is Holder continuous of order $1 / 2$.

Lemma 8.2. Suppose that Condition 2.2 holds. Let $\kappa>0$. Then, there exists a constant $C_{\kappa}$ depending on $\kappa$ such that for all $X, Y \in \mathbf{R}^{2}, x, y>0$ and $t \in \mathbf{R}_{+}$,

$$
\begin{array}{r}
\left|\sigma_{\kappa}\left(t, e^{X}\right)-\sigma_{\kappa}\left(t, e^{Y}\right)\right| \leq C_{\kappa}|X-Y| \\
\left|\sigma_{\kappa}(t, x)-\sigma_{\kappa}(t, y)\right| \leq C_{\kappa} \sqrt{|x-y|}
\end{array}
$$

Proof.

Using the assumption and the fact that the mapping $X \mapsto e^{X} \wedge \kappa$ is Lipschitz, we get that

$$
\begin{aligned}
\left|\sigma_{\kappa}\left(t, e^{X}\right)-\sigma_{\kappa}\left(t, e^{Y}\right)\right| & =\left|\sigma_{\kappa}\left(t, e^{X} \wedge \kappa\right)-\sigma_{\kappa}\left(t, e^{Y} \wedge \kappa\right)\right| \\
& \leq C_{\kappa}\left|e^{X} \wedge \kappa-e^{Y} \wedge \kappa\right| \leq C_{\kappa}|X-Y|
\end{aligned}
$$


On the other hand, we have

$$
\begin{aligned}
\left|\sigma_{\kappa}(t, x)-\sigma_{\kappa}(t, y)\right| & =\left|\sigma_{\kappa}(t, x \wedge \kappa)-\sigma_{\kappa}(t, y \wedge \kappa)\right|, \\
& \leq C_{\kappa}|x \wedge \kappa-y \wedge \kappa| \leq C_{\kappa} \sqrt{|x \wedge \kappa-y \wedge \kappa|}, \\
& \leq C_{\kappa} \sqrt{|x-y|} .
\end{aligned}
$$

The last inequalities hold since $\sqrt{|x \wedge \kappa-y \wedge \kappa|}$ is bounded by $\sqrt{2 \kappa}$ and the mapping $x \mapsto x \wedge \kappa$ is Lipschitz.

Proof of Lemma 3.1. Suppose that Condition 2.2 holds. Consider the sequence of stopping times

$$
\tau^{\kappa}:=\inf \left\{t: S_{t} \geq \kappa\right\}
$$

Since the stopped processes $S^{\tau^{\kappa}}$ and $\left(S_{\kappa}\right)^{\tau^{\kappa}}$ satisfy the same s.d.e., we deduce that $S_{t}=S_{\kappa}(t)$ on $t \in\left[0, \tau^{\kappa}\right]$. As $\tau^{\kappa} \rightarrow \infty$, we then conclude. Under Condition 2.3, we follow the same arguments with

$$
\tau^{\kappa}:=\inf \left\{t: S_{t} \leq \kappa^{-1}\right\}
$$

Note that $\tau^{\kappa} \rightarrow \tau$ as $\kappa \rightarrow \infty$.

Lemma 8.3. Assume that Condition 3.3 (I) is satisfied. Then, there exists a constant $C$ independent of $\kappa$ such that $\sup _{u \leq T} \mathbf{E} S_{\kappa}(u) \leq C$.

Proof. By definition, we have $S_{\kappa}(u) / F_{u}\left(r_{\kappa}\left(\cdot, S_{\kappa}(\cdot)\right)\right) \leq M_{u}$ where $M_{0}=1$ and $M$ is a local martingale which is solution to the s.d.e.

$$
d M_{u}=M_{u} \sigma_{\kappa}\left(u, S_{\kappa}(u)\right) d W_{u}
$$

As $r$ is supposed to be bounded, we deduce that $S_{\kappa}(u) \leq C S_{0} M_{u}$. Let $\left(\tau^{n}\right)$ be a sequence of stopping times such that $M^{\tau^{n}}$ is a true martingale. Then, $\mathbf{E} S_{\kappa}\left(u \wedge \tau^{n}\right) \leq C S_{0}$ and finally $\mathbf{E} S_{\kappa}(u) \leq C S_{0}$ by virtue of by Fatou's lemma.

Lemma 8.4. Assume that Assumption 3.3 (I) is satisfied. Then, for all $p$ such that $p \leq 0$ or $p \geq 1 / 2$, there exists a constant $C_{p}$ independent of $\kappa$ such that $\sup _{u \leq T} \mathbf{E}\left(S_{\kappa}(u)\right)^{p} \leq C_{p}$. 
Proof. Since $r$ is bounded, the following inequality holds for all fixed $q \geq 0$ :

$$
\left(S_{\kappa}(u)\right)^{p} \leq C_{q} S_{0}^{p} N_{u} e^{\frac{1}{2}(q-p) \int_{0}^{u} \sigma_{\kappa}^{2}\left(r, S_{\kappa}(r)\right) d r}
$$

where

$$
N_{u}:=\exp \left\{p \int_{0}^{u} \sigma_{\kappa}\left(r, S_{\kappa}(r)\right) d W_{r}-\frac{1}{2} q \int_{0}^{u} \sigma_{\kappa}^{2}\left(r, S_{\kappa}(r)\right) d r\right\} .
$$

Using the inequality $0 \leq a b \leq\left(a^{2}+b^{2}\right)$, we get that

$$
\left(S_{\kappa}(u)\right)^{p} \leq C S_{0}^{p} \tilde{N}_{u}+C S_{0}^{p} e^{(q-p) \int_{0}^{u} \sigma^{2}\left(r, S_{\kappa}(r) \wedge \kappa\right) d r},
$$

where $\tilde{N}=N^{2}$ is a local martingale if we choose $q=2 p^{2}$. Moreover, as the function $x \mapsto e^{(q-p) u x}$ is convex, the Jensen inequality and the hypothesis yields that

$$
\begin{aligned}
e^{(q-p) \int_{0}^{u} \sigma^{2}\left(r, S_{\kappa}(r) \wedge \kappa\right) d r} & \leq \frac{1}{u} \int_{0}^{u} e^{(q-p) r \sigma^{2}\left(r, S_{\kappa}(r) \wedge \kappa\right)} d r \\
& \leq C_{p}+\frac{C}{u} \int_{0}^{u}\left(\left(\log \left(S_{\kappa}(r)\right)\right)^{k(q-p) T} 1_{S_{\kappa}(r) \wedge \kappa \geq 1}+1_{S_{\kappa}(r) \wedge \kappa \leq 1}\right) d r
\end{aligned}
$$

where $k$ is a constant. Using the property

$$
(\log (x \vee 1))^{k(q-p) T} \leq C_{p} x, \quad \forall x \geq 0,
$$

and Lemma 8.3, we deduce that $E\left(S_{\kappa}(u)\right)^{p} \leq C_{p}$.

Lemma 8.5. Assume that Condition 3.3 (II) is satisfied. Then, for all $p$ such that $p \geq 0$, there exists a constant $C_{p}$ independent of $\kappa$ such that $\sup _{u \leq T} \mathbf{E}\left(S_{\kappa}(u)\right)^{p} \leq C_{p}$.

Proof. Let us consider $N=N(p) \in \mathbf{N}$ such that $0 \leq p \leq 2 N \alpha$. Observe that, for $p \geq 0,0 \leq\left(S_{\kappa}(u)\right)^{p} \leq 1+\left(S_{\kappa}(u)\right)^{2 N \alpha} 1_{S_{\kappa}(u) \geq 1} \leq 1+\left(S_{\kappa}(u)\right)^{2 N \alpha}$. Therefore, it is enough to show the lemma when $p=2 N \alpha$. We show by induction that the statement holds for every $p=2 j \alpha, 0 \leq j \leq N$. This is trivial if $j=0$. Applying the Ito formula when $q=2(j+1) \alpha, j \geq 0$, we get that

$$
\begin{aligned}
\left(S_{\kappa}(u)\right)^{q}= & \left(S_{0}\right)^{q}+\int_{0}^{u} q\left(S_{\kappa}(r)\right)^{q} \sigma_{\kappa}\left(r, S_{\kappa}(r)\right) d W_{r}+\int_{0}^{r} q\left(S_{\kappa}(r)\right)^{q} r_{\kappa}\left(r, S_{\kappa}(r)\right) d r \\
& +\frac{1}{2} q(q-1) \int_{0}^{u}\left(S_{\kappa}(r)\right)^{q} \sigma_{\kappa}^{2}\left(r, S_{\kappa}(r)\right)(r) d r .
\end{aligned}
$$


Recall that $\sigma_{\kappa}$ is bounded. Using the Doleans-Dade representation of $S_{\kappa}(u)$, we deduce that $S_{\kappa}(u)$ admits finite moments at any order. It follows that the Ito integral in the inequality above is a square integrable martingale. Therefore,

$$
\begin{aligned}
\mathbf{E}\left(S_{\kappa}(u)\right)^{q}= & \left(S_{0}\right)^{q}+\int_{0}^{u} q \mathbf{E}\left(S_{\kappa}(r)\right)^{q} r_{\kappa}\left(r, S_{\kappa}(r)\right) d r \\
& +\frac{1}{2} q(q-1) \int_{0}^{u} \mathbf{E}\left(S_{\kappa}(r)\right)^{q} \sigma_{\kappa}^{2}\left(r, S_{\kappa}(r)\right) d r .
\end{aligned}
$$

We then use the inequality $r \leq 1+r^{2}$ and by Assumption 3.3 (II), we deduce that $g^{j+1}(u):=\mathbf{E}\left(S_{\kappa}(u)\right)^{2(j+1) \alpha}$ satisfies

$$
\begin{aligned}
g^{j+1}(u) \leq & \max _{j \leq N}\left(S_{0}\right)^{2(j+1) \alpha}+C\left(N^{2} \alpha^{2}+N \alpha\right) \int_{0}^{u} g^{j}(r) d r \\
& +C\left(N \alpha+N^{2} \alpha^{2}\right) \int_{0}^{u} g^{j+1}(r) d r
\end{aligned}
$$

Suppose that $g^{j} \leq C^{j}$ where $C^{j}$ does not depend on $\kappa$. Then, by Gronwall's lemma, we deduce that $g^{j+1} \leq C^{j+1}$ where $C^{j+1}$ does not depend on $\kappa$. The conclusion follows with $j=N$ since the number of steps $N(p)$ is finite and only depends on $p$.

Corollary 8.6. Assume that Condition 3.3 is satisfied. Then, for all $p \geq 0$, there exists a constant $C_{p}$ independent of $\kappa$ such that $\mathbf{E}_{\sup _{u \leq T}}\left(S_{\kappa}(u)\right)^{p} \leq C_{p}$.

Proof. Since $\|\cdot\|_{L^{p_{1}}} \leq\|\cdot\|_{L^{p_{2}}}$ if $1 \leq p_{1} \leq p_{2}$, and $\left(S_{\kappa}(u)\right)^{p} \leq 1+S_{\kappa}(u)$ when $p \in[0,1]$, we may assume without loss of generality that $p \in 2 \mathbb{N}$. Let us first suppose that Condition (I) holds. We recall that

$$
S_{\kappa}(t)=S_{0}+\int_{0}^{t} \sigma\left(u, S_{\kappa}(u) \wedge \kappa\right) S_{\kappa}(u) d W_{u}+\int_{0}^{t} r\left(u, S_{\kappa}(u) \wedge \kappa\right) S_{\kappa}(u) d u .
$$

Since $0 \leq \sigma^{2}(t, x) \leq C(1+x)$ and $r$ is bounded, we easily deduce that $E \sup _{u \leq T}\left(S_{\kappa}(u)\right)^{p} \leq C_{p}$ by the Burkholder-Davis-Gundy and Jensen inequalities as well as Lemma 8.4.

Let us now suppose that Condition (II) holds. In this case, $0 \leq S_{\kappa}^{p}(u)\left(\sigma^{p}\left(u, S_{\kappa}(u) \vee \kappa^{-1}\right)+r^{p}\left(u, S_{\kappa}(u) \vee \kappa^{-1}\right)\right) \leq C\left(S_{\kappa}^{p}(u)+S_{\kappa}^{(1-\alpha) p}(u)\right)$ 
Since $1-\alpha \geq 0$, we use Lemma 8.5 and conclude as previously.

Since $S_{\kappa}$ converges pointwise on $[0, \tau]$ to $S, S=S_{\kappa}$ on $\left[0, \tau^{\kappa}\right]$ where $\tau^{\kappa} \rightarrow \tau$, and $S_{t}=0$ on $t \geq \tau$, we apply Fatou's Lemma and deduce the following.

Corollary 8.7. Assume that Condition 3.3 is satisfied. Then, for all $p \geq 0$, there exists a constant $C$ such that $E \sup _{u \leq T}\left(S_{u}\right)^{p} \leq C_{p}$.

Remark 8.8. Recall that

$$
d Y_{t}=\sigma\left(t, S_{t}\right) d W_{t}+r\left(t, S_{t}\right) d t-\frac{1}{2} \sigma^{2}\left(t, S_{t}\right) d t
$$

Under Condition $3.3(I)$, since $0 \leq \sigma^{2}(t, x) \leq C(1+x)$, we then deduce that $E \sup _{u \leq T} Y_{u}^{2}<\infty$ as the coefficient $r$ is bounded. In particular, the process $\int_{0}^{\cdot} \sigma\left(t, S_{t}\right) d W_{t}$ is a true martingale.

Proof of Lemma 3.6. If $\sigma_{\kappa}=\sigma(\cdot, \wedge \kappa)$, we consider the stopping time $\tau^{k}$ defined by (8.11). We obtain that

$$
\begin{aligned}
\mathbf{E} \sup _{t \in[0, T]}\left|S_{\kappa}(t)-S_{t}\right|^{p} & =\mathbf{E} \sup _{t \in[0, T]}\left|S_{\kappa}(t)-S_{t}\right|^{p} 1_{\tau^{\kappa}<t} \\
& \leq \mathbf{E} \sup _{t \in[0, T]}\left|S_{\kappa}(t)-S_{t}\right|^{p} 1_{\tau^{\kappa}<T} .
\end{aligned}
$$

In the case where $\tau^{k}$ is defined by (8.11), we deduce that

$$
\begin{aligned}
\mathbf{E} \sup _{t \in[0, T]}\left|S_{\kappa}(t)-S_{t}\right|^{p} & \leq \mathbf{E} \sup _{t \in[0, T]}\left|S_{\kappa}(t)-S_{t}\right|^{p} 1_{\sup _{t \in[0, T]} S_{t} \geq \kappa} \\
& \leq \sqrt{\mathbf{E} \sup _{t \in[0, T]}\left|S_{\kappa}(t)-S_{t}\right|^{2 p}} \sqrt{\frac{\mathbf{E}\left(\sup _{t \in[0, T]} S_{t}\right)^{2 l}}{\kappa^{2 l}}}
\end{aligned}
$$

The conclusion follows. In the case where $\sigma_{\kappa}=\sigma\left(\cdot, \cdot \vee \kappa^{-1}\right)$, let $\tau^{k}$ be defined by (8.12). Then,

$$
\begin{aligned}
\mathbf{E} \sup _{t \in[0, \tau \wedge T]}\left|S_{\kappa}(t)-S_{t}\right|^{p}= & \mathbf{E} \sup _{t \in[0, T]}\left|S_{\kappa}(t)-S_{t}\right|^{p} 1_{\tau^{\kappa}<t \leq \tau \wedge T} \\
\leq & \mathbf{E} \sup _{t \in[0, T]}\left|S_{\kappa}(t)-S_{t}\right|^{p} 1_{\tau^{\kappa}<t \leq \tau} 1_{\tau \leq T} \\
& +\mathbf{E} \sup _{t \in[0, T]}\left|S_{\kappa}(t)-S_{t}\right|^{p} 1_{\inf }{ }_{t \in[0, T]} S_{t} \leq \kappa^{-1} 1_{\tau>T} .
\end{aligned}
$$


Notice that the second term of the last inequality above is bounded as follows:

$$
\mathbf{E} \sup _{t \in[0, T]}\left|S_{\kappa}(t)-S_{t}\right|^{p} 1_{\inf _{t \in[0, T]} S_{t} \leq \kappa^{-1}} 1_{\tau>T} \leq \sqrt{\mathbf{E} \sup _{t \in[0, T]}\left|S_{\kappa}(t)-S_{t}\right|^{2 p}} \gamma(\tau, \kappa),
$$

where $\gamma(\tau, \kappa):=P\left(\inf _{t \in[0, T]} S_{t} \leq \kappa^{-1} ; \tau>T\right) \rightarrow 0$ as $\kappa \rightarrow \infty$. If $t \in\left[\tau^{\kappa}, \tau\right]$, where $\tau<T$, then

$$
\begin{aligned}
S_{\kappa}(t) & =\kappa^{-1}+\int_{\tau^{\kappa}}^{t} S_{\kappa}(u) \sigma_{\kappa}\left(u, S_{\kappa}(u)\right) d W_{u}+\int_{\tau^{\kappa}}^{t} S_{\kappa}(u) r_{\kappa}\left(u, S_{\kappa}(u)\right) d u \\
S_{t} & =\kappa^{-1}+\int_{\tau^{\kappa}}^{t} S(u) \sigma(u, S(u)) d W_{u}+\int_{\tau^{\kappa}}^{t} S(u) r(u, S(u)) d u .
\end{aligned}
$$

Under Condition (II) of Condition 3.3, we have

$$
\begin{aligned}
\left|S_{\kappa}(u) \sigma_{\kappa}\left(u, S_{\kappa}(u)\right)\right|+\left|S_{\kappa}(u) r_{\kappa}\left(u, S_{\kappa}(u)\right)\right| & \leq C\left(\left|S_{\kappa}(u)\right|+\left|S_{\kappa}(u)\right|^{1-\alpha}\right) \\
\left|S_{\kappa}(u) \sigma_{\kappa}\left(u, S_{\kappa}(u)\right)\right|+\left|S_{\kappa}(u) r_{\kappa}\left(u, S_{\kappa}(u)\right)\right| & \leq C\left(\left|S_{\kappa}(u)\right|+\left|S_{\kappa}(u)\right|^{1-\alpha}\right) .
\end{aligned}
$$

Using the Burkholder-Davis-Gundy inequalities and the Cauchy-Schwarz inequality, we deduce that the term $\delta(\tau, \kappa)=\mathbf{E}_{\sup _{t \in[0, T]}}\left|S_{\kappa}(t)-S_{t}\right|^{p} 1_{\tau^{\kappa}<t<\tau} 1_{\tau \leq T}$ is bounded as follows:

$\delta(\tau, \kappa) \leq C\left(\sup _{\kappa} \sqrt{\mathbf{E} \sup _{t \in[0, T]}\left|S_{\kappa}(t)\right|^{2 p}}+\sqrt{\mathbf{E} \sup _{t \in[0, T]}|S(t)|^{2 p}}\right) \sqrt{\mathbf{E}\left|\tau^{\kappa} \wedge T-\tau \wedge T\right|^{p}}$.

By the Lebesgue theorem, since $\tau^{\kappa} \rightarrow \tau$, we deduce that $\mathbf{E}\left|\tau^{\kappa} \wedge T-\tau \wedge T\right|^{p} \rightarrow 0$ as $\kappa \rightarrow \infty$. The conclusion follows.

Lemma 8.9. Let $\left(g^{m}\right)_{m}$ be a sequence of positive functions defined on an interval $[0, T], T>0$, such that for some $C>0$, we have:

$$
g^{m+1}(t) \leq C \int_{0}^{t} g^{m}(u) d u, \quad 0 \leq g^{0} \leq C
$$

Then

$$
\sup _{t \in[0, T]} g^{m}(t) \leq C^{m} \frac{t^{m}}{m !} \sup _{t \in[0, T]} g^{0}(t) .
$$

Proof. The proof relies on the following recursive reasoning. Set

$$
C_{g}:=\sup _{t \in[0, T]} g^{0}(t) \leq C
$$


Suppose that

Then, we have

$$
\sup _{t \in[0, T]} g^{n-1}(t) \leq C^{n-1} \frac{t^{n-1}}{(n-1) !} C_{g}
$$

$$
g^{n}(t) \leq C \int_{0}^{t} C^{n-1} \frac{u^{n-1}}{(n-1) !} C_{g} d u .
$$

The claim follows.

Proof of Lemma 3.7. If $\alpha \geq 2$, recall that for all $x, y \geq 0$ :

$$
\left|x^{\alpha / 2}-y^{\alpha / 2}\right| \leq \frac{\alpha}{2}(\max (|x| ;|y|))^{\alpha / 2-1}|x-y| .
$$

We deduce by Conditions 2.2 and 2.3 that

$$
\left(\int_{0}^{t} \sigma_{\kappa, m+1}^{2}(s)-\sigma_{\kappa, m}^{2}(s) d s\right)^{2} \leq C C_{\kappa} \int_{0}^{t}\left(\sigma_{\kappa, m+1}(s)-\sigma_{\kappa, m}(s)\right)^{2} d s,
$$

by virtue of Jensen's inequality. Together with the Burkholder-Davis-Gundy inequalities, we obtain that

$$
\begin{aligned}
\mathbf{E} \sup _{u \leq t}\left|Y_{\kappa, m+1}(u)-Y_{\kappa, m}(u)\right|^{2} & \leq C C_{\kappa} \mathbf{E} \int_{0}^{t} \sup _{u \leq s}\left|\sigma_{\kappa, m+1}(u)-\sigma_{\kappa, m}(u)\right|^{2} d s \\
& +C C_{\kappa} \mathbf{E} \int_{0}^{t} \sup _{u \leq s}\left|r_{\kappa, m+1}(u)-r_{\kappa, m}(u)\right| d s .
\end{aligned}
$$

By Conditions 2.2 or 2.3, with Lemma 8.2,

$$
\left|\sigma_{\kappa}\left(t, e^{X}\right)-\sigma\left(t, e^{Y}\right)\right|+\left|r_{\kappa}\left(t, e^{X}\right)-r\left(t, e^{Y}\right)\right| \leq C_{\kappa}|X-Y|
$$

hence

$$
\left|\sigma_{\kappa, m+1}(u)-\sigma_{\kappa, m+1}(u)\right|+\left|r_{\kappa, m+1}(u)-r_{\kappa, m+1}(u)\right| \leq C_{\kappa}\left|Y_{\kappa, m}(u)-Y_{\kappa, m-1}(u)\right| .
$$

We deduce that

$$
\mathbf{E} \sup _{u \leq t}\left|Y_{\kappa, m+1}(u)-Y_{\kappa, m}(u)\right|^{2} \leq C_{\kappa} \int_{0}^{t} \mathbf{E} \sup _{u \leq s}\left|Y_{\kappa, m}(u)-Y_{\kappa, m-1}(u)\right|^{2} d s,
$$

where $C_{\kappa}$ is a constant depending on $\kappa$. Using Lemma 8.9, we get that

$$
\begin{aligned}
\mathbf{E} \sup _{u \leq t}\left|Y_{\kappa, m+1}(u)-Y_{\kappa, m}(u)\right|^{2} & \leq\left(C_{\kappa}\right)^{m+1} \mathbf{E} \sup _{u \leq t}\left|Y_{\kappa, 1}(u)-Y_{\kappa, 0}(u)\right|^{2} \frac{t^{m}}{m !}, \\
& \leq C\left(C_{\kappa}\right)^{m+1} \frac{t^{m+1}}{(m+1) !}\left(1+S_{0}^{2}\right),
\end{aligned}
$$

where $C$ does not depend on $m$ and $\kappa$. We deduce Inequality (3.6). Similarly, we obtain Inequality (3.7) and the claim follows. 
Lemma 8.10. Let $a_{1}, \cdots a_{k}$ be real numbers. we have the following inequality

$$
\left(\sum_{l=1}^{k} a_{l}\right)^{2} \leq \sum_{l=1}^{k} 2^{l} a_{l}^{2}, \quad\left(\sum_{l=1}^{k} a_{l}\right)^{4} \leq \sum_{l=1}^{k} 8^{l} a_{l}^{4}
$$

Proof. For any real numbers $a, b$, the inequality $2 a b \leq a^{2}+b^{2}$ implies that $(a+b)^{2} \leq 2 a^{2}+2 b^{2}$. Therefore, $(a+b)^{4} \leq\left(2 a^{2}+2 b^{2}\right)^{2} \leq 8 a^{4}+8 b^{4}$. We conclude by induction.

Proof of Corollary 3.8. Using Lemma 8.10, we obtain the inequality

$$
\mathbf{E} \sup _{u \leq t}\left|Y_{\kappa, \infty}(u)-Y_{\kappa, m}(u)\right|^{2} \leq \sum_{j=m}^{\infty} 2^{j} \mathbf{E} \sup _{u \leq t}\left|Y_{\kappa, j+1}(u)-Y_{\kappa, j}(u)\right|^{2} .
$$

We deduce the first inequality from (8.13) and, similarly, the second one. The conclusion follows.

Proof of Lemma 3.9. Applying the Burkholder-Davis-Gundy and Jensen inequalities, we get that

$$
\begin{aligned}
\mathbf{E} \sup _{u \in[0, t]}\left(S_{\kappa, m+1}(u)-S_{\kappa, m}(u)\right)^{2} \leq & C \mathbf{E} \int_{0}^{t}\left(S_{\kappa, m+1}(s) \sigma_{\kappa, m+1}(s)-S_{\kappa, m}(s) \sigma_{\kappa, m}(s)\right)^{2} d s, \\
& +2 \mathbf{E} \int_{0}^{t}\left(S_{\kappa, m+1}(s) r_{\kappa, m+1}(s)-S_{\kappa, m}(s) r_{\kappa, m}(s)\right)^{2} d s,
\end{aligned}
$$

where we recall that $\sigma_{\kappa, m}(s):=\sigma_{\kappa}\left(s, S_{\kappa, m-1}(s)\right)$. The first term in the r.h.s. of the inequality is bounded from above as follows:

$$
\begin{aligned}
& \mathbf{E} \int_{0}^{t}\left(S_{\kappa, m+1}(s) \sigma_{\kappa, m+1}(s)-S_{\kappa, m}(s) \sigma_{\kappa, m}(s)\right)^{2} d s \leq \\
& 2 \mathbf{E} \int_{0}^{T} S_{\kappa, m+1}^{2}(s)\left[\sigma_{\kappa, m+1}(s)-\sigma_{\kappa, m}(s)\right]^{2} d s+2 \mathbf{E} \int_{0}^{T}\left(\sigma_{m, \kappa}(s)\left(S_{\kappa, m+1}(s)-S_{\kappa, m}(s)\right)\right)^{2} d s .
\end{aligned}
$$

The second one is similarly bounded. Recall the inequalities $\left|\sigma_{\kappa}\right|+\left|r_{\kappa}\right| \leq C_{\kappa}$, $\kappa>0$, and recall that, by assumption or by Lemma 8.2,

$$
\left|\sigma_{\kappa}\left(t, e^{X}\right)-\sigma_{\kappa}\left(t, e^{Y}\right)\right|+\left|r_{\kappa}\left(t, e^{X}\right)-r_{\kappa}\left(t, e^{Y}\right)\right| \leq C_{\kappa}|X-Y| .
$$

Moreover, using Gronwall's lemma, since $\sigma_{\kappa}$ and $r_{\kappa}$ are bounded by a constant depending on $\kappa$, we deduce the existence of $C_{p, \kappa}>0$ such that for all $p$

$$
\sup _{m} \mathbf{E} \sup _{t \in[0, T]} S_{\kappa, m}^{2 p}(t) \leq C_{p, \kappa}
$$


Using the stochastic integral representation of $S_{\kappa, m+1}$ and $S_{\kappa, m}$ respectively, we obtain by means of Cauchy-Schwarz's inequality, Jensen's inequality and the Ito isometry that

$$
\begin{aligned}
\operatorname{Esup}_{u \in[0, t]}\left(S_{\kappa, m+1}(u)-S_{\kappa, m}(u)\right)^{2} \leq & \quad C_{\kappa} \sqrt{\mathbf{E} \sup _{u \leq T}\left|Y_{\kappa, m}(u)-Y_{\kappa, m-1}(u)\right|^{4}} \\
+ & C_{\kappa} \int_{0}^{t} \mathbf{E} \sup _{u \leq s}\left(S_{\kappa, m+1}(u)-S_{\kappa, m}(u)\right)^{2} d s .
\end{aligned}
$$

Using Gronwall's Lemma, we deduce that

$$
\mathbf{E} \sup _{t \in[0, T]}\left(S_{\kappa, m+1}(t)-S_{\kappa, m}(t)\right)^{2} \leq C_{\kappa} \sqrt{\mathbf{E} \sup _{t \leq T}\left|Y_{\kappa, m}(t)-Y_{\kappa, m-1}(t)\right|^{4}}
$$

The conclusion follows.

Proof of Lemma 3.10. By virtue of Lemma 8.10, we get the inequality

$$
\begin{aligned}
\mathbf{E} \sup _{t \in[0, T]}\left(S_{\kappa, \infty}(t)-S_{\kappa, m}(t)\right)^{2} & \leq \sum_{j=m}^{\infty} 2^{j} \mathbf{E} \sup _{u \in[0, t]}\left(S_{\kappa, j+1}(u)-S_{\kappa, j}(u)\right)^{2} \\
& \leq C_{\kappa} \sum_{j=m}^{\infty} \frac{\left(C_{\kappa}\right)^{j}}{\sqrt{j !}}
\end{aligned}
$$

Proof of Lemma 3.11. Since $\sigma_{\kappa}$ and $r_{\kappa}$ are bounded by a constant depending on $\kappa$, we deduce by the Burkholder-Davis-Gundy inequalities and the Gronwall's lemma a constant $c_{\kappa}>0$ such that for all $m, n$,

$$
\mathbf{E} \sup _{t \in[0, T]}\left|S_{\kappa, m}(t)+S_{\kappa, m, n}(t)\right|^{4} \leq c_{\kappa} .
$$

For a sake of simplicity, we suppose that $r=0$ as the proof is similar for the general case. Therefore,

$$
\begin{aligned}
S_{\kappa, 1}(t) & =S_{0}+\int_{0}^{t} S_{\kappa, 1}(u) \sigma_{\kappa}\left(u, S_{0}\right) d W_{u} \\
S_{\kappa, 1, n}(t) & =S_{0}+\int_{0}^{t} S_{\kappa, 1, n}(u) \sigma_{\kappa}\left(u^{(n)}, S_{0}\right) d W_{u} .
\end{aligned}
$$

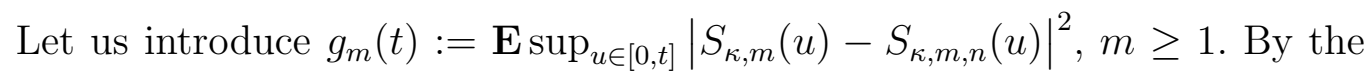


Doob's inequality,

$$
\begin{aligned}
g_{1}(t) & \leq 4 \int_{0}^{t}\left(S_{\kappa, 1, n}(u) \sigma_{\kappa}\left(u^{(n)}, S_{0}\right)-S_{\kappa, 1}(u) \sigma_{\kappa}\left(u, S_{0}\right)\right)^{2} d u \\
g_{1}(t) & \leq 8 \int_{0}^{t} \sigma_{\kappa}^{2}\left(u^{(n)}, S_{0}\right)\left(S_{\kappa, 1, n}(u)-S_{\kappa, 1}(u)\right)^{2} d u \\
& +8 \int_{0}^{t} S_{\kappa, 1}^{2}(u)\left(\sigma_{\kappa}\left(u^{(n)}, S_{0}\right)-\sigma_{\kappa}\left(u, S_{0}\right)\right)^{2} d u
\end{aligned}
$$

Since $\sigma_{\kappa}$ is bounded by a constant $C_{\kappa}$ and $\left|\sigma_{\kappa}\left(u^{(n)}, S_{0}\right)-\sigma_{\kappa}\left(u, S_{0}\right)\right| \leq T / n$, we deduce by (8.15) that

$$
g_{1}(t) \leq \frac{C_{\kappa}}{n^{2}}+C_{\kappa} \int_{0}^{t} g(u) d u, \quad t \in[0, T] .
$$

By the Gronwall's lemma, we deduce that $g_{1}(T) \leq C_{\kappa} / n^{2}$ for some constant $C_{\kappa}$ which does not depend on $n$. More generally, if $m \geq 2$, we have

$$
\begin{aligned}
S_{\kappa, m}(t) & =S_{0}+\int_{0}^{t} S_{\kappa, m}(u) \sigma_{\kappa}\left(u, S_{\kappa, m-1}(u)\right) d W_{u}, \\
S_{\kappa, m, n}(t) & =S_{0}+\int_{0}^{t} S_{\kappa, m, n}(u) \sigma_{\kappa}\left(u^{(n)}, S_{\kappa, m-1, n}^{(n)}(u)\right) d W_{u} .
\end{aligned}
$$

Therefore, by the Doob's inequality,

$$
\begin{aligned}
g_{m}(t) & \leq 4 \int_{0}^{t}\left(S_{\kappa, m, n}(u) \sigma_{\kappa}\left(u^{(n)}, S_{\kappa, m-1, n}^{(n)}(u)\right)-S_{\kappa, m}(u) \sigma_{\kappa}\left(u, S_{\kappa, m-1}(u)\right)\right)^{2} d u \\
g_{m}(t) & \leq 8 \int_{0}^{t} \sigma_{\kappa}^{2}\left(u^{(n)}, S_{\kappa, m-1, n}^{(n)}(u)\right)\left(S_{\kappa, m, n}(u)-S_{\kappa, m}(u)\right)^{2} d u \\
& +8 \int_{0}^{t} S_{\kappa, m}^{2}(u)\left(\sigma_{\kappa}\left(u^{(n)}, S_{\kappa, m-1, n}^{(n)}(u)\right)-\sigma_{\kappa}\left(u, S_{\kappa, m-1, n}(u)\right)\right)^{2} d u \\
& +8 \int_{0}^{t} S_{\kappa, m}^{2}(u)\left(\sigma_{\kappa}\left(u, S_{\kappa, m-1, n}(u)\right)-\sigma_{\kappa}\left(u, S_{\kappa, m-1}(u)\right)\right)^{2} d u .
\end{aligned}
$$

Recall that $\sigma_{\kappa}$ is bounded by a constant $C_{\kappa}$ and

$$
\left|\sigma_{\kappa}\left(u^{(n)}, S_{\kappa, m-1, n}(u)\right)-\sigma_{\kappa}\left(u, S_{\kappa, m-1, n}(u)\right)\right| \leq C_{\kappa} / n
$$

Therefore, we may obtain upper bounds for the two first terms of the r.h.s. in the inequality above as we do for $m=1$. Precisely, we also need to estimate $\mathbf{E}\left(S_{\kappa, m-1, n}^{(n)}(u)-S_{\kappa, m-1, n}(u)\right)^{2}$. To do so, it suffices to recall that 
$\mathbf{E s u p}_{t \in[[0, T]]} S_{\kappa ; m}^{4}(t) \leq C_{\kappa}$ for some constant $C_{\kappa}$ which does not depend on $m$ hence, if $u \in\left[t_{i}^{n}, t_{i+1}^{n}\right), i=0, \cdots, n$, we deduce by the Ito isometry and the Cauchy-Schwarz inequality that $\mathbf{E}\left(S_{\kappa, m-1, n}^{(n)}(u)-S_{\kappa, m-1, n}(u)\right)^{2} \leq C_{\kappa} n^{-1}$ where $C_{\kappa}$ only depends on $\kappa$.

At last, an upper bound for the third term is obtained using the CauchySchwarz inequality, (8.15) and the following inequality (see Lemmas 8.2 and 8.1):

$$
\mid \sigma_{\kappa}\left(u, S_{\kappa, m-1, n}(u)\right)-\sigma_{\kappa}\left(u, S_{\kappa, m-1}(u) \mid \leq C_{\kappa} \sqrt{\left|S_{\kappa, m-1, n}(u)-S_{\kappa, m-1}(u)\right|} .\right.
$$

Therefore,

$$
g_{m}(t) \leq \frac{C_{\kappa}}{n}+C_{\kappa} g_{m-1}(t)+C_{\kappa} \int_{0}^{T} g_{m}(u) d u .
$$

By the Gronwall's lemma, we then deduce a constant $C_{\kappa}$ which only depends on $\kappa$ such that

$$
g_{m}(T) \leq \frac{C_{\kappa}}{n}+C_{\kappa} g_{m-1}(T)
$$

By induction, we deduce that, for all $m \geq 2$,

$$
g_{m}(T) \leq n^{-1} \sum_{j=1}^{m}\left(C_{\kappa}\right)^{j} \leq \alpha_{\kappa}\left(C_{\kappa}\right)^{m} n^{-1}
$$

for some constant $\alpha_{\kappa}$.

Lemma 8.11. Let $S$ be a positive stochastic process such that $P\left(\inf _{t \in[0, T]} S_{t}=\right.$ $a)=0$ for all $a \in\left(0, S_{0}\right)$. Let $\left(S^{n}\right)_{n \in \mathbb{N}}$ be a sequence of stochastic pro-

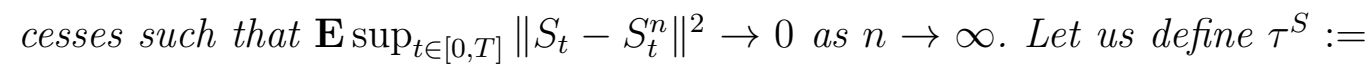
$\inf \left\{t: S_{t}<a\right\}$ and $\tau^{S^{n}}:=\inf \left\{t: S_{t}^{n}<a\right\}$. Then, $\mathbf{E}\left|1_{\tau^{S}>T}-1_{\tau^{S^{n}}>T}\right| \rightarrow 0$ as $n \rightarrow \infty$.

Proof. Suppose that $\mathbf{E}\left|1_{\tau^{S}>T}-1_{\tau^{S^{n}}>T}\right| \rightarrow l>0$ for a subsequence. Since $\left|\inf _{t \in[0, T]} S_{t}-\inf _{t \in[0, T]} S_{t}^{n}\right| \leq \sup _{t \in[0, T]}\left\|S_{t}-S_{t}^{n}\right\|$, we may also suppose that $\inf _{t \in[0, T]} S_{t}^{n}$ converges to $\inf _{t \in[0, T]} S_{t}$ a.s. for a common subsequence. In the case where $\tau>T$, i.e. $\inf _{t \in[0, T]} S_{t} \geq a$, as $P\left(\inf _{t \in[0, T]} S_{t}=a\right)=0$, we may assume that $\inf _{t \in[0, T]} S_{t}>a$ hence $\inf _{t \in[0, T]} S_{t}^{n}>a$ for $n$ large enough and, finally $\tau^{S^{n}}>T$. If $\tau \leq T$, $\inf _{t \in[0, T]} S_{t}^{n}<a$ and then, similarly, we obtain that 
$\tau^{S^{n}} \leq T$ for $n$ large enough. We deduce that $\left|1_{\tau^{S}>T}-1_{\tau^{S^{n}}>T}\right| \rightarrow 0$ a.s. in contradiction with $l>0$.

Lemma 8.12. Let $S$ be a positive stochastic process such that $P\left(\inf _{t \in[0, T]} S_{t}=\right.$ $a)=0$ for all $a>0$. Let $\left(S_{n}\right)_{n \in \mathbb{N}}$ be a sequence of stochastic processes such that $\mathbf{E} \sup _{t \in[0, T]}\left|S_{t}-S_{n}(t)\right|^{2} \rightarrow 0$ as $n \rightarrow \infty$. Let us denote by $S_{n}^{(n)}$ its discrete version given in Section 2.1. Let us define $\tau^{S}:=\inf \left\{t: S_{t}<a\right\}$ and $\tau^{S_{n}^{(n)}}:=\inf \left\{t: S_{n}^{(n)}(t)<a\right\}$. Then, $\mathbf{E}\left|1_{\tau^{S}>T}-1_{\tau^{S_{n}^{(n)}}>T}\right| \rightarrow 0$.

Proof. Recall that $\tau^{S}>T$ if and only if $\inf _{t \in[0, T]} S_{t} \geq a$. Let us first show that, for any random interval $I \subseteq[0, T], \sup _{t \in I} S_{n}(t)$ converges in $L^{2}$ to $\sup _{t \in I} S_{t}$ as $n \rightarrow \infty$. To see it, note that for all $t \in[0, T], S_{t} \leq S_{n}(t)+\sup _{t \in[0, T]}\left|S_{t}-S_{n}(t)\right|$ hence $\sup _{t \in I} S_{t} \leq \sup _{t \in I} S_{n}(t)+\sup _{t \in[0, T]}\left|S_{t}-S_{n}(n)\right|$. By symmetry, we get that

$$
\left|\sup _{t \in I} S_{n}(t)-\sup _{t \in I} S_{t}\right| \leq \sup _{t \in[0, T]}\left|S_{t}-S_{n}(t)\right|
$$

and the conclusion follows. Note that the same property holds if we replace the infinimum by the supremum of $S$ over $I$.

On the set $\left\{\inf _{t \in[0, T]} S_{t}<a\right\}$, as $S$ is continuous, we may construct by a measurable selection argument a measurable interval $I=\left[t_{0}-\varepsilon, t_{0}+\varepsilon\right]$, $\varepsilon>0$ a.s., such that $\sup _{t \in I} S_{t}<a$. Otherwise, we arbitrarily set $I=[0, T]$.

Suppose that for a subsequence $\mathbf{E}\left|1_{\tau^{S}>T}-1_{\tau^{S_{n}^{(n)}}>T}\right| \rightarrow l>0$. Consider another subsequence such that we also have $\sup _{t \in[0, T]}\left|S_{t}-S_{n}(t)\right| \rightarrow 0$ a.s. By the first step for the infinimum, if $\inf _{t \in[0, T]} S_{t}>a$, then $\inf _{t \in[0, T]} S_{n}(t)>a$ if $n$ is large enough. As $\inf _{t \in[0, T]} S_{n}^{(n)}(t) \geq \inf _{t \in[0, T]} S_{n}(t)$, we deduce that $\inf _{t \in[0, T]} S_{n}^{(n)}(t)>a$ and, finally, $\left|1_{\tau^{S}>T}-1_{\tau^{S_{n}^{(n)}}>T}\right|=0$ if $n$ is large enough.

On the set $\inf _{t \in[0, T]} S_{t}<a$, i.e. when $\tau^{S} \leq T$, we have $\sup _{t \in I} S_{t}<a$. By the first step, $\sup _{t \in I} S_{n}(t)<a$ if $n$ is large enough. Choose $n$ sufficiently large so that $T / n<\varepsilon(\omega)$. Then, the random discrete date $t_{i}^{n}$ such that $t_{0}(\omega) \in$ $\left[t_{i}^{n}, t_{i+1}^{n}\right)$ satisfies $t_{i}^{n} \in I$ hence $S_{n}^{(n)}\left(t_{0}\right)=S_{n}\left(t_{i}^{n}\right)<a$. It follows that $\tau^{S_{n}^{(n)}} \leq T$. We finally deduce by the Lebesgue theorem that $\mathbf{E}\left|1_{\tau^{S}>T}-1_{\tau^{S_{n}^{(n)}}>T}\right| \rightarrow 0$ in contradiction with $l>0$.

Lemma 8.13. Consider the case where $\sigma_{\kappa}=\sigma\left(\cdot, \cdot \vee \kappa^{-1}\right)$ and $r_{\kappa}=r(\cdot, \cdot \vee$ 
$\left.\kappa^{-1}\right)$ under Condition 2.3 with $P(\tau \leq T)>0$. The random variables $Z_{\kappa}=$ $\inf _{t \in[0, T]} Y_{\kappa}(t), \kappa>0$, and $Z_{\kappa, m}=\inf _{t \in[0, T]} Y_{\kappa, m}(t), m \geq 1$, admit a density with respect to the Lebesgue measure.

Proof. The proof is based on [19][Proposition 2.1.11]. To do so, we need verify the conditions of [19][Proposition 2.1.10]. As the mapping $h(x)=x \vee \kappa^{-1}$ is not differentiable at point $\kappa^{-1}$, we first approximate $h$ by

$$
\begin{aligned}
h^{n}(x) & =\kappa^{-1} 1_{\left(-\infty, \kappa^{-1}-n^{-1}\right]}(x)+x 1_{\left[\kappa^{-1}+n^{-1}, \infty\right)}(x) \\
& +\left(\kappa^{-1}+\frac{n}{4}\left(x-\kappa^{-1}+n^{-1}\right)^{2}\right) 1_{\left[\kappa^{-1}-n^{-1}, K+n^{-1}\right]}(x)
\end{aligned}
$$

We have the following properties:

$$
0 \leq h^{n}(x)-h(x) \leq \frac{1}{4 n}, \quad\left|\left(h^{n}\right)^{\prime}(x)-h^{\prime}(x)\right| \leq 1_{\left[\kappa^{-1}-n^{-1}, \kappa^{-1}+n^{-1}\right]}(x) .
$$

We then consider the solution $Y_{\kappa}^{n}$ of the s.d.e. (3.4) where we replace $\sigma_{\kappa}$ by

$$
\sigma_{\kappa}^{n}(t, x):=\sigma\left(t, h^{n}(x)\right)
$$

We may show that $\sup _{t \in[0, T]}\left|Y_{\kappa}^{n}(t)-Y_{\kappa}(t)\right| \rightarrow 0$ in $L^{2}$ as $n \rightarrow \infty$. Therefore, since $Y_{\kappa}^{n}$ admits a derivative in the Malliavin sense, we also deduce that $Y_{\kappa} \in \mathrm{D}^{1,2}$. Moreover, for all $s \leq t$, making $n \rightarrow \infty$, we get that

$$
\begin{aligned}
\mathrm{D}_{s} Y_{\kappa}(t) & =\sigma_{\kappa}\left(s, S_{0} e^{Y_{\kappa}(s)}\right)+\int_{s}^{t} \mathrm{D}_{s} Y_{\kappa}(u) h^{\prime}\left(S_{0} e^{Y_{\kappa}(u)}\right) S_{0} e^{Y_{\kappa}(u)} \nabla_{x} \sigma_{\kappa}\left(t, S_{0} e^{Y_{\kappa}(u)}\right) d W_{u}, \\
& +\int_{s}^{t} \mathrm{D}_{s} Y_{\kappa}(u) h^{\prime}\left(S_{0} e^{Y_{\kappa}(u)}\right) S_{0} e^{Y_{\kappa}(u)} \nabla_{x} \gamma_{\kappa}\left(t, S_{0} e^{Y_{\kappa}(u)}\right) d u
\end{aligned}
$$

where $\gamma_{\kappa}=r_{\kappa}-\sigma_{\kappa}^{2} / 2$. By the assumptions, e.g. $X \nabla_{X} \sigma(t, X)$ is bounded if $X \geq \kappa^{-1}$ and $h^{\prime}(X)=0$ if $X \leq \kappa^{-1}$, and the Gronwall's lemma, we deduce that

$$
\mathbf{E} \sup _{t \in[0, T]}\left|\mathrm{D}_{s} Y_{\kappa}(t)\right|^{p}<\infty
$$

whatever $p \geq 2$. By the Ito formula, we obtain an expression of $\left[\mathrm{D}_{s} Y_{\kappa}(t)\right]^{2}$ for $t \geq s$ as a stochastic integral. By the stochastic Fubini theorem, we then also deduce a representation of $t \mapsto \int_{0}^{t}\left[\mathrm{D}_{s} Y_{\kappa}(t)\right]^{2} d s$ as a stochastic integral. Using the Gronwall's lemma, we finally deduce that $\mathbf{E}_{\sup _{t \leq T}} \int_{0}^{t}\left[\mathrm{D}_{s} Y_{\kappa}(t)\right]^{2} d s<\infty$, 
i.e. the conditions of [19][Proposition 2.1.10] hold. Moreover, as $\left(\mathrm{D}_{s} Y_{\kappa}(t)\right)_{t \geq s}$ is a Doléans-Dade exponential, it is strictly positive and we finally deduce that [19][Proposition 2.1.11] applies, i.e. $Z_{\kappa}=\inf _{t \in[0, T]} Y_{\kappa}(t)$ admits a density with respect to the Lebesgue measure. The second statement is shown similarly by induction for $m=0,1, \cdots$.

Proof of Theorem 3.12. Notice that we have

$$
\begin{aligned}
\left|\mathbf{E} G(S) 1_{\tau>T}-\mathbf{E} G\left(S_{\kappa, 1, n}^{(n)}\right) 1_{\tilde{\tau}^{\kappa, 1, n}>T}\right| \leq & \mathbf{E}\left|G(S)-G\left(S_{\kappa}\right)\right| 1_{\tau>T} \\
& +\mathbf{E}\left|G\left(S_{\kappa}\right)-G\left(S_{\kappa, m}\right)\right| \\
& +\mathbf{E}\left|G\left(S_{\kappa, m}\right)-G\left(S_{\kappa, m, n}\right)\right| \\
& +\mathbf{E} \mid G\left(S_{\kappa, m, n}\right)-G\left(S_{\kappa, m, n}^{(n)}\right) \\
& +\mathbf{E}|G(S)|\left|1_{\tau>T}-1_{\tilde{\tau}^{\kappa, 1, n}>T}\right|,
\end{aligned}
$$

Since $G$ is supposed to be Lipschitz, the first term (8.16) is bounded from above by $\varepsilon_{\kappa}$ as stated in Lemma 3.6. The second term (8.17) is bounded from above by the error $\varepsilon_{\kappa, m}$ given by Lemma 3.10 . The term (8.18) is bounded from above according to Lemma 3.11. The consistency property with respect to discretization satisfied by $G$ allows us to estimate the fourth term (8.19). As to the last term, notice that $G$ is of linear growth since it is Lipschitz. By Corollary 8.7, we deduce from the Cauchy-Schwarz inequality a constant $C$ such that

$$
\mathbf{E}|G(S)|\left|1_{\tau>T}-1_{\tilde{\tau}^{\kappa, 1, n}>T}\right| \leq C \sqrt{\mathbf{E}\left|1_{\tau>T}-1_{\tilde{\tau}^{\kappa, 1, n}>T}\right|}
$$

It remains to show that $\mathbf{E}\left|1_{\tau>T}-1_{\tilde{\tau}^{\kappa, m, n}>T}\right| \rightarrow 0$ a.s. as $\kappa, m, n \rightarrow \infty$. Let us introduce $\tilde{\tau}^{\kappa}:=\inf \left\{t: S_{\kappa}(t)<\kappa^{-1}\right\}$. Since $S$ and $S_{\kappa}$ coincides on $\left[0, \tau^{\kappa}\right]$ and $\tilde{\tau}^{\kappa}=\tau^{\kappa}>T$ if $\kappa$ is large enough and $\tau>T$, we deduce that $\left|1_{\tau>T}-1_{\tilde{\tau}^{\kappa}>T}\right| \rightarrow$ 0 if $\tau>T$ as $\kappa \rightarrow \infty$. If $\tau \leq T$, we have $\tau^{\kappa} \leq \tau$ so that $\left|1_{\tau>T}-1_{\tau^{\kappa}>T}\right|=$ 0 . Therefore, $\mathbf{E}\left|1_{\tau>T}-1_{\tilde{\tau}^{\kappa}>T}\right| \rightarrow 0$ as $\kappa \rightarrow+\infty$. It remains to show that $\mathbf{E}\left|1_{\tilde{\tau}^{\kappa}>T}-1_{\tilde{\tau}^{\kappa, m, n}>T}\right| \rightarrow 0$ a.s. as $\kappa, m, n \rightarrow \infty$. Let us introduce $\tilde{\tau}^{\kappa, m}:=$ $\inf \left\{t: S_{\kappa, m}(t)<\kappa^{-1}\right\}$. By Proposition 3.10, Lemma 8.13 and Lemma 8.11, for fixed $\kappa, \mathbf{E}\left|1_{\tilde{\tau}^{\kappa}>T}-1_{\tilde{\tau}^{\kappa, m}>T}\right| \rightarrow 0$ as $m \rightarrow \infty$. Therefore, it remains to show that $\mathbf{E}\left|1_{\tilde{\tau}^{\kappa, m}>T}-1_{\tilde{\tau}^{\kappa, m, n}>T}\right| \rightarrow 0$ a.s. as $n \rightarrow \infty$. By virtue of Lemma 3.11, Lemma 8.13 and Lemma 8.12, E $\left|1_{\tilde{\tau}^{\kappa, m}>T}-1_{\tilde{\tau}^{\kappa, m, n}>T}\right| \rightarrow 0$ a.s. as $n \rightarrow \infty$. 


\section{References}

[1] Black F., Scholes M. The pricing of options and corporate liabilities. The Journal of Political Economy, 81 , 637-659 (1973).

[2] Bouleau N., Lépingle D. Numerical methods for stochastic processes. John Wiley and Sons Inc.(1994).

[3] Brigo D., Mercurio F. A mixed-up smile. Risk, 13(9), 123-126 (2000).

[4] Dana R.A., Jeanblanc Monique. Financial markets in continuous time. Springer-Verlag Berlin Heidelberg (2003).

[5] Delbaen F., Shirakawa H. A note on option pricing for constant elasticity of variance model. Asia-Pacific Financial Markets, 2, 2, 85-99(2002).

[6] Desmond J. H., Xuerong M. Convergence, non-negativity and stability of a new Milstein scheme with applications to finance. Discrete and continuous dynamical systems, series B, 18, 8, 2083-2100 (2013).

[7] Dupire B. Pricing and hedging with smiles. Mathematics of Derivative Securities. Cambridge Univ. Press, 15, 103-111 (1997).

[8] Friedman A. Stochastic Differential Equations And Applications. Dover Books on Mathematics (1975).

[9] Funahashi H. A chaos expansion approach under hybrid volatility models. Quantitative Finance, 14, 11, 1923-1936 (2014).

[10] Giles Michael B. Improved multilevel Monte Carlo convergence using the Milstein scheme. Monte Carlo and quasi-Monte Carlo methods, 343-358, Springer, Berlin 2008 (2006).

[11] Hull J., White A. The pricing of options on assets with stochastic volatilities. Journal of Finance, 42, 281-300(1987).

[12] Hutzenthaler M., Jentzen A. On a perturbation theory and on strong convergence rates for stochastic ordinary and partial differential equations with non-globally monotone coefficients. arXiv:1401.0295, 1$41(2014)$.

[13] Hutzenthaler M., Jentzen A. and Kloeden Peter E. Strong and weak divergence in finite time of Eulers method for stochastic differential equations with non-globally Lipschitz continuous coefficients. Proc. R. Soc. Lond. Ser. A Math. Phys. Eng. Sci., 467:1563-1576 (2011). 
[14] Hutzenthaler M., Jentzen A., and Kloeden Peter E. Divergence of the multilevel Monte Carlo Euler method for nonlinear stochastic differential equations. Ann. Appl. Probab., 23(5):1913171966 (2013).

[15] Neuenkirch A. and Szpruch L. First order strong approximations of scalar SDEs defined in a domain. Numerische Mathematik, 1-34 (2014).

[16] Kijima M., Funahashi H. A chaos expansion approach for the pricing of contingent claims. Journal of Computational Finance, 18, 3, 27-58 (2015).

[17] Kloeden P. E., Platen E. Numerical solution of stochastic differential equations. Springer-Verlag Berlin Heidelberg, Stochastic Modelling and Applied Probability $(23,1992)$.

[18] Lindsay A.E., Brecher D.R. Simulation of the CEV process and the local martingale property. Mathematics and Computers in Simulation, 82, 5, 86817878 (2012).

[19] Nualart D. The Malliavin Calculus and Related Topics. Probability, its applications (2006). Springer Berlin Heidelberg.

[20] Mehrdoust F., Babaei S. and Fallah S. Efficient Monte Carlo option pricing under CEV model. Communications in Statistics - Simulation and Computation, DOI 10.1080/03610918.2015.1040497 (2015).

[21] Schroder M. Computing the constant elasticity of variance option pricing formula. The Journal of Finance, 44, 1, 211-219 (1989). 